\title{
A dislocation density based elasto-plastic self-consistent model for the prediction of cyclic deformation: Application to AA6022-T4
}

\author{
Milovan Zecevic and Marko Knezevic ${ }^{*}$ \\ Department of Mechanical Engineering, University of New Hampshire, Durham, NH 03824, USA
}

\begin{abstract}
We develop a polycrystal plasticity constitutive law based on the elasto-plastic self-consistent (EPSC) theory for the prediction of cyclic tension-compression deformation. The crystallography based model integrates a dislocation based hardening model and accounts for inter-granular stresses and slip system level backstresses, which make it capable of capturing non-linear unloading and the Bauschinger effect (BE). Furthermore, the model features dissolution of dislocation population upon the load reversal, which enables it to predict the change in hardening rate during reverse loading from that during forward loading. To demonstrate these capabilities of the model, we investigate elasto-plastic behavior of AA6022-T4 sheets under in-plane cyclic tension-compression. From a set of carefully performed cyclic tests to several strain levels, we observe that the material exhibits (1) a typical decreasing hardening rate during forward loading, (2) a linear followed by non-linear unloading upon the load reversal, (3) a transient softening followed by rapid work hardening (the $\mathrm{BE}$ ), and (4) a decrease in subsequent hardening rate during reverse loading (the permanent softening phenomenon). To predict these effects, we calibrate the model to establish a set of material parameters using a portion of the measured data. The remaining measured data is used for verification of the model. We show that using the single set of material parameters, the developed model is capable of predicting all the particularities pertaining to the cyclic deformation of the material with great accuracy. From the favorable comparison of the predictions and experimental data, we infer first that the non-linearity of unloading increases with the amount of pre-strain, next that the backstresses have a dominant effect in capturing non-linear unloading while both the backstresses and inter-granular stresses govern the $\mathrm{BE}$, and finally that the inclusion of reversible dislocation motion is the key for capturing hardening rates during reverse loading.
\end{abstract}

Keywords: B. Bauschinger effect; B. Residual stress; B. Backstress; A. Dislocations; B. Crystal plasticity

\footnotetext{
* Corresponding author at: University of New Hampshire, Department of Mechanical Engineering, 33 Academic Way, Kingsbury Hall, W119, Durham, New Hampshire 03824, United States. Tel.: 603862 5179; fax: 603862 1865.

E-mail address: marko.knezevic@unh.edu (M. Knezevic).
} 


\section{Introduction}

Numerical simulations play a major role in optimization of metal forming processes and evaluation of component performances in service. Accuracy of such simulations is highly dependent on the accuracy of a material model, which is integral in such numerical tools. Since metal forming processes involve non-monotonic and multi-axial deformation paths, it is necessary for the material model to be sensitive to such deformation path changes. For example, the springback behavior of forming parts after removal from a die depends on the elasto-plastic behavior of the material during unloading (Boger et al., 2005; Wagoner et al., 2013). The bending-unbending behavior of sheets drawn over a die radius depends on the elasto-plastic behavior of the material during tension followed by compression (Chun et al., 2002; Wagoner et al., 2013).

In addition to the flow stress and concomitant work hardening rate during forward monotonic loading, material models sensitive to strain reversals must capture the following directional effects: (1) linear and non-linear unloading, (2) transient in yield stress and work hardening rate immediately after re-yielding known as the Bauschinger effect (BE), and (3) subsequent work hardening rate, which is usually different from that during the forward loading. These effects are attributed to the evolution of the underlying physical phenomena and the microstructure, which are briefly summarized below.

Non-linear unloading behavior refers to a small departure from a linear elastic material law during unloading (Wagoner et al., 2013; Yoshida et al., 2002). The total unloading strain comprises an initial elastic component followed by small scale re-yielding attributed to a dislocation relaxation phenomenon (Mompiou et al., 2012; Pavlina et al., 2015; Sritharan and Chandel, 1997; Yoshida et al., 2002; Zhou and LeSar, 2012). This small scale back flow during unloading is a consequence of the presence of micro backstresses formed during forward loading. These micro backstresses arise from dislocation pile-ups, which consist of dislocations incorporated into grain boundaries during forward loading. Upon unloading, the micro backstresses relax, giving rise to a micro plastic strain in the reverse direction. This micro plastic strain is accommodated by a partial re-emission of the dislocations from the grain boundary pile-ups (Mompiou et al., 2012; Sritharan and Chandel, 1997). Level of plastic deformation before unloading is expected to influence the magnitude of deviation from the linear unloading behavior. Therefore, capturing the effect of non-linear unloading by constitutive models requires modeling of prior plastic deformation.

The BE (Bauschinger, 1886) refers to the change in yield strength followed by rapid hardening of a material deformed in tension (compression) and subsequently in compression (tension). The BE effect has been observed in the deformation of polycrystals (Nieh and Nix, 1986; Sauzay, 2008; Stout and Rollett, 1990) as well as single crystals (Buckley and Entwistle, 1956; Demir and Raabe, 2010; Gough et al., 1927; Greetham and Honeycombe, 1960) and its phenomenology and microstructural descriptions are well documented (Abel, 1987; Bate and Wilson, 1986a). Similar to non-linear unloading, it arises from the local deviation from an applied stress due to backstresses having inter- and intra-granular sources. The intra-granular backstress sources explain the BE in single crystals. In contrast to nonlinear unloading of polycrystals, dislocation grain boundary pile-ups cannot be the origin of 
these backstresses in single crystals. Incompatibility between hard dislocation walls of high dislocation density separated by soft regions of low dislocation density develops long-range internal stresses (LRIS) (aka intra-granular stresses or type III stresses) (Kassner et al., 2013; Mughrabi, 1983). During forward deformation LRIS are induced in the opposite direction from that of the applied stress. Upon reloading, LRIS combine with the reversed loading stress, causing a drop in the onset of plastic deformation. Orowan's theory, which states that there is an anisotropy in resistance to dislocation motion between forward and reverse motion, offers an additional explanation of the BE in single crystals. Dislocations move easier in the reversed direction because obstacles on the same path have been overcome during the forward motion (Orowan, 1959). The BE effect in polycrystals is caused by that in single crystals, by the existence of additional sources of backstresses, and by inter-granular stresses (type II stresses). The additional sources of backstresses are the pile-ups at grain boundaries (Seeger, 1957; Weng, 1979) and any non-deforming particles (Brown and Clarke, 1975; Tanaka and Mori, 1970). Depending on the crystallographic orientation with respect to the loading axis, the yield strengths of different grains are divergent with some grains being harder and some being softer. The inter-granular stresses build up due to strain incompatibility between adjacent hard/soft grains of different crystal orientations during forward loading. Similarly these stresses assist the stress applied in the reverse direction, which is reflected in the drop of the yield stress (Weng, 1979, 1980). Therefore, both intraand inter-granular stresses give rise to the $\mathrm{BE}$ in polycrystals. These stresses are strongly linked to the concurrent evolutions of crystallographic and morphological texture with plastic strain, which induce the plastic anisotropy.

Following the $\mathrm{BE}$, a change of the work hardening rate occurs during reverse loading to large strains (Hasegawa et al., 1975a; Kitayama et al., 2013; Stout and Rollett, 1990; Wilson et al., 1990). This change manifests in an offset between the forward and the reverse flow stresses (Bate and Wilson, 1986b; Orowan, 1959; Zang et al., 2013) and is referred to as permanent softening. The effect originates from the annihilation/dissolution of loosely tangled dislocation contained in dislocation substructures formed during the primary deformation path, as well as slow buildup of new dislocation substructures during deformation in the opposite direction.

Incorporation of the above summarized effects in constitutive models represents a challenging task. Clearly, simple phenomenological material models based on isotropic continuum plasticity are insufficient ( $\mathrm{Li}$ et al., 2002). A range of more sophisticated phenomenological models have been developed to quantitatively predict the BE through a combination of isotropic and linear or non-linear kinematic hardening laws (Armstrong and Frederick, 1966; Chaboche and Rousselier, 1983; Chaboche, 1977; Chaboche, 2008; Hu et al., 1992; McDowell, 1992). These phenomenological models, while computationally efficient and relatively easy to implement within commercial finite element codes, are not physically based and therefore do not directly account for the mechanistic sources of backstresses, or even distinguish between inter- and intra-granular contributions. A significant limitation of these models is that it is difficult to find the values of the material parameters, demanding expensive and complex mechanical tests and inverse methodologies 
to find the parameters (Smith et al., 2014). Additionally, these models apply to a specific initial material state and to the specific loading conditions used in the model fitting process.

An attractive alternative is the physics-based crystal plasticity theory, which is based on the crystallography of various deformation mechanisms and considers crystallographic texture as an input state variable that evolves with plastic strain. Such physical description of plastic deformation naturally incorporates development of the plastic anisotropy caused by textural and microstructural changes during deformation (Lopes et al., 1999). Polycrystal plasticity models link response of constituent single crystals to the response of a polycrystalline aggregate. For this purpose different homogenization schemes exist ranging from an upperbound Taylor (Al-Harbi et al., 2010; Fromm et al., 2009; Kalidindi et al., 2009; Knezevic et al., 2009; Knezevic et al., 2008a; Knezevic et al., 2008b; Knezevic and Savage, 2014; Mihaila et al., 2014; Shaffer et al., 2010; Taylor, 1938; Wu et al., 2007), to lower-bound Sachs (Fast et al., 2008; Knezevic and Kalidindi, 2007; Sachs, 1929), to mean-filed selfconsistent (Lebensohn and Tomé, 1993), and finally to full-field finite element models (Kalidindi et al., 1992; Kalidindi et al., 2006; Knezevic et al., 2014c; Knezevic et al., 2013c; Knezevic et al., 2010). To facilitate simulations under heterogeneous plastic strains, these polycrystal codes are further coupled with commercial finite element codes to operate at a FE integration point (Jahedi et al., 2015a; Jahedi et al., 2015b; Jahedi et al., 2014; Knezevic et al., 2014d; Knezevic et al., 2013d; Zecevic et al., 2015b, c). Polycrystal plasticity models intrinsically account for anisotropic elasto-plastic behavior of the material and, depending on the selected homogenizations, are capable of calculating the inter-granular sources of backstress. While the Taylor homogenization predicts large inter-granular stresses, the Sachs model develops no such stresses. Though finite element homogenization would lead to the most accurate estimates of backstresses, it is computationally impractical. Self-consistent homogenization provides the best compromise between computational speed and accuracy. In addition to predictions of mechanical response, the added benefits of polycrystal plasticity models include their ability to provide valuable information about texture evolution as well as physical insights into active deformation modes. Moreover, these models are not constrained to an initial material state or to a specific deformation path, and therefore are much more predictive and robust than macroscopic plasticity models.

The elasto-plastic self-consistent (EPSC) model (Turner and Tomé, 1994) is among the most widely applied crystal plasticity models for the prediction of inter-granular stresses. However, the inherent inter-granular stresses predicted by EPSC were found insufficient to capture subtleties of cyclic deformation of stainless steel (Lorentzen et al., 2002). The primary reason for the poor predictions is lack of backstress kinematic hardening effects. More recently, backstresses at the slip system level have been incorporated within the Voce hardening law in EPSC, resulting in successful predictions of small strain cyclic deformation (Wollmershauser et al., 2012). Since this model was implemented within the phenomenological Voce hardening law, it has been unable to capture subsequent hardening behavior during reloading to large strains as a function of the level of pre-loading strain.

On the other hand, the visco-plastic self-consistent (VPSC) model (Lebensohn and Tomé, 1993) was used with an extended Voce hardening law for modeling the BE (Beyerlein and 
Tomé, 2007) and with a dislocation based hardening law for modeling both the BE and subsequent work hardening rates to large strains upon strain reversal (Kitayama et al., 2013; Knezevic et al., 2013a; Wen et al.). The key feature of the later model is that it includes a reversible dislocation population that annihilates upon strain reversal, enabling reverse hardening rate predictions. The backstresses in VPSC cannot be entirely related to physical phenomena, since VPSC neglects effect of inter-granular stresses. Moreover, due to the absence of elasticity VPSC cannot model the unloading effects.

Taking advantages of the above developments in EPSC and VPSC, we present a comprehensive dislocation based hardening law in EPSC for cyclic deformation. The law is directional at the slip system level and evolves the slip resistance and internal stresses with microstructure rearrangements upon load reversal. Specifically, it accounts for the intergranular stresses and the backstress kinematic hardening at the slip system level for the predictions of non-linear unloading and the subsequent BE upon reloading. The model considers dislocation dissolution for the prediction of hardening rates during loading in the reverse direction. To demonstrate predictive capabilities of the model, we characterize the cyclic tension-compression response of the AA6022-T4 alloy and use the dataset for calibration and verification. We show that the new hardening law within the EPSC polycrystal model is capable of capturing all the particularities associated with the cyclic response of the material. Since the microstructural features are incorporated in the hardening law, the model successfully predicts the cyclic material behavior as a function of loading directions and level of pre-strain. We found that the non-linearity of unloading increases with the amount of pre-strain; the backstresses control the non-linear unloading while both the backstresses and inter-granular stresses govern the $\mathrm{BE}$; and that the reversible dislocation motion plays the major role in hardening during reverse loading.

The paper is organized as follows. Cyclic tension-compression measured data and characterization data of the initial microstructure of AA6022-T4 are presented in section 2. The new EPSC model is described in section 3. Results of the cyclic simulations and role of the individual effects through the comparisons between simulated and measured cyclic curves are presented in section 4 . Conclusions are presented in section 5.

\section{Material characterization and cyclic tension-compression measurements}

The material investigated here is a sheet of 6022 heat treatable low copper, Al-Si-Mg alloy (Alcoa). This widely used alloy offers an excellent combination of strength and formability. The material was in the temper T4 condition (AA6022-T4). Figure 1a shows an orientation map collected using the electron backscattered diffraction (EBSD) orientation imaging method to depict the grain structure of the alloy. The average grain size in the material is approximately $50 \mu \mathrm{m}$ with grains slightly elongated in the rolling direction (RD). To acquire information about the initial crystallographic texture in the material, multiple EBSD scans were collected over an area of several square millimeters. Figure $1 \mathrm{~b}$ shows the corresponding pole figures. Presence of a strong cube texture component is evident from the pole figures. 


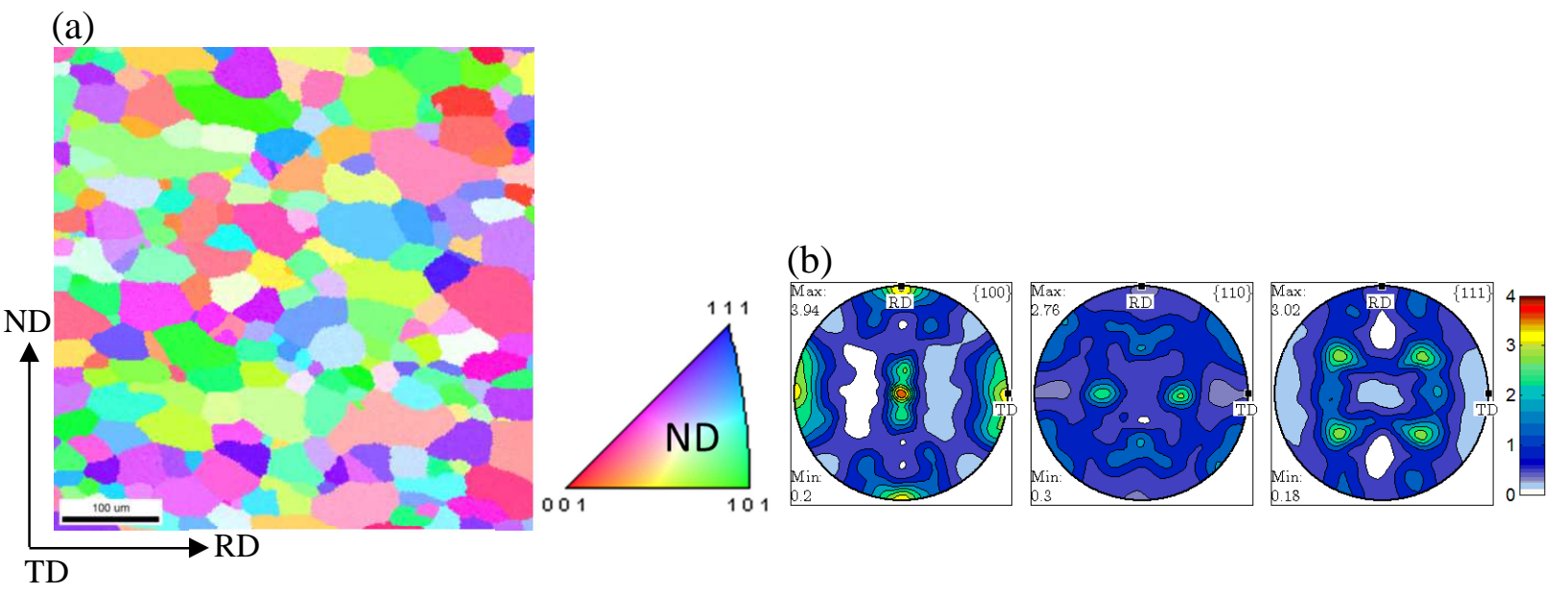

Fig. 1. (a) Orientation map and (b) pole figures of the initial microstructure and texture of the AA6022-T4 sheet.

The focus of the present paper is to develop a constitutive law sensitive to strain reversals. Various experimental methods have been developed to test materials under such conditions including forward and reverse torsion (Chen et al., 1999; Stout and Rollett, 1990), forward and reverse simple shear (Gracio et al., 2004), tension followed by torsion (Brown, 1970), tension followed by simple shear (Barlat et al., 2003; Peeters et al., 2001b), and tension followed by compression (Boger et al., 2005; Dietrich and Turski, 1978; Szczepiński, 1990; Tan et al., 1994; Yoshida et al., 2002). Here, the AA6022-T4 alloy was tested under the cycle tension-compression to several strain levels.

Specimens were cut out of sheets of AA6022-T4 with orientations along the rolling direction (RD) and along the transverse direction (TD). For cyclic tension-compression, the specimens were $130 \mathrm{~mm}$ long and $12.5 \mathrm{~mm}$ wide with a $12.5 \mathrm{~mm}$ gauge length by $10 \mathrm{~mm}$ in width. To make sure that the tension portion of the cyclic tension-compression curves matches regular tension test data, additional tensile specimens were made and tested. Both the cyclic tensioncompression and tension tests were performed on the MTS machine 858 Table Top System. A set of specialized fixtures were used for the cyclic tests as described in (Dietrich and Turski, 1978; Szczepiński, 1990). Cyclic tests at strain rate of $0.004 \mathrm{~s}^{-1}$ were performed to $2 \%$ strain for the RD and TD samples and to $5 \%$ and $10 \%$ for the RD samples.

Figure 2 depicts several representations of the measured true stress-true strain curves including the normalized strain hardening rate plots. It is observed that the alloy exhibits a classical decreasing hardening rate throughout the response in tension, which is typical for materials in which the plastic deformation is accommodated by crystallographic slip. Upon unloading, the material exhibits an initial linear portion and a subsequent non-linear portion of unloading behavior (Fig. 2a and b). The non-linear portion of the unloading curve is a function of plastic strain, and it increases with strain (Fig. 2a). Figure 2c, d and e depict the hardening behavior. The overall decrease of the hardening rate during the reverse loading in compression is more pronounced than forward loading and features two stages. In the first stage, the strain hardening rate recovers the level before unloading within a relatively small amount of strain. Subsequently, the strain hardening rate decreases faster than it did during 
the forward loading (Fig. 2d). As a result, the flow stress exhibits permanent softening (Fig. 2c). This type of permanent softening after strain reversal was first observed in (Hasegawa et al., 1975a) for a polycrystalline Al alloy during tension and compression loading and more recently for the AA6022-T4 alloy during forward and reverse simple shear loading in (Gracio et al., 2004). This softening was attributed to the annihilation of dislocations (recovery effects) after the reversal of the slip directions during loading in the reverse direction. The difference in the normalized strain hardening behavior in tension vs. compression is clearly evident from Fig. 2e. The normalization factor is the shear modulus, $\mu$, taken to be $26.1 \mathrm{GPa}$ (Meyers M.A., 2009). The two curves start from the value corresponding to the ratio between Young's modulus and the shear modulus, $E / \mu$. Due to non-linear unloading, the compressive curve exhibits drop in strain-hardening rates at zero macroscopic stress. Evidently, the hardening rate is steeper during tensile loading.

Our objective here is to introduce these effects in a crystallographic hardening model which is, in turn, implemented into the polycrystalline EPSC model. The modeling framework is described in the next section.
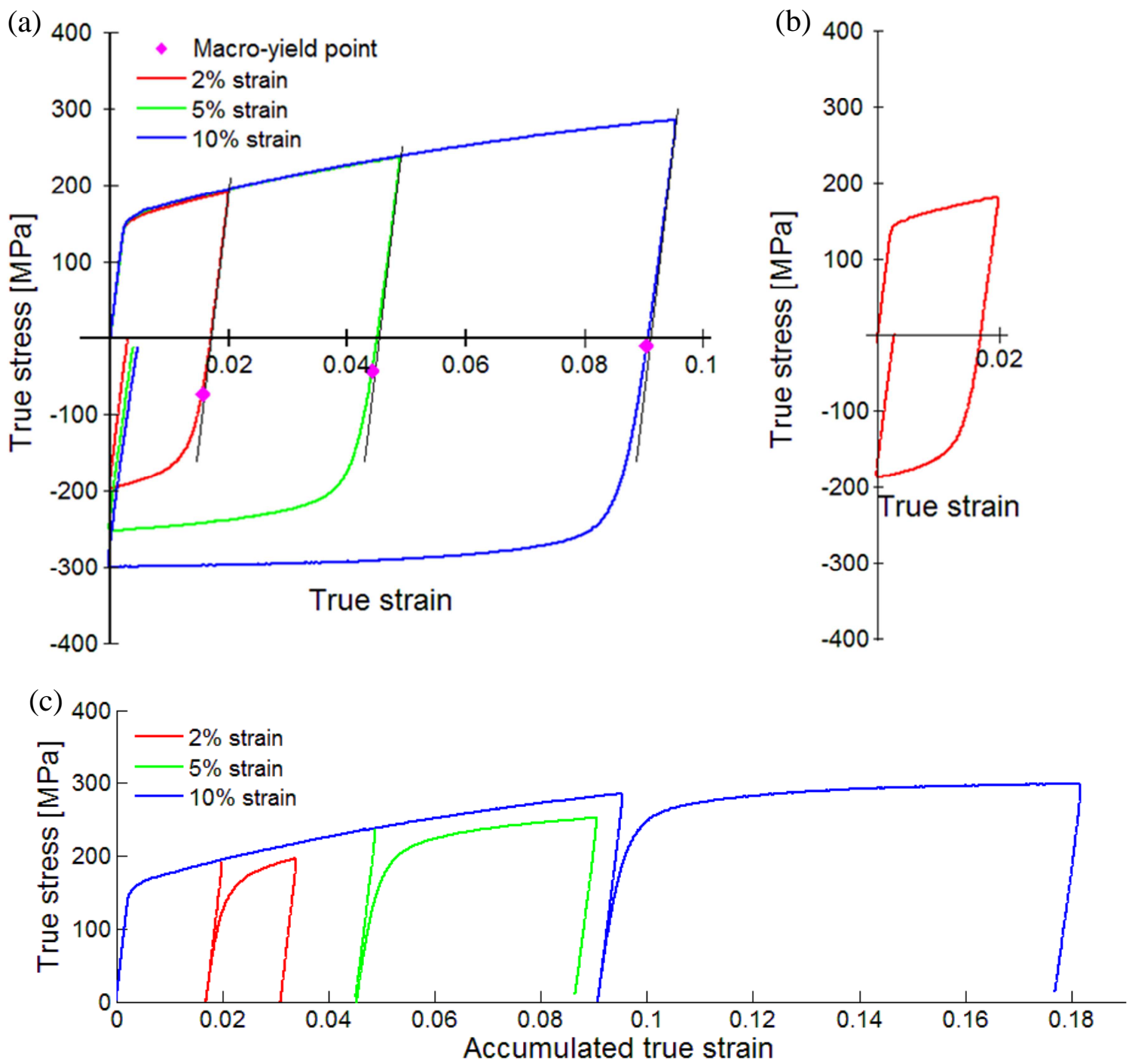
(d)

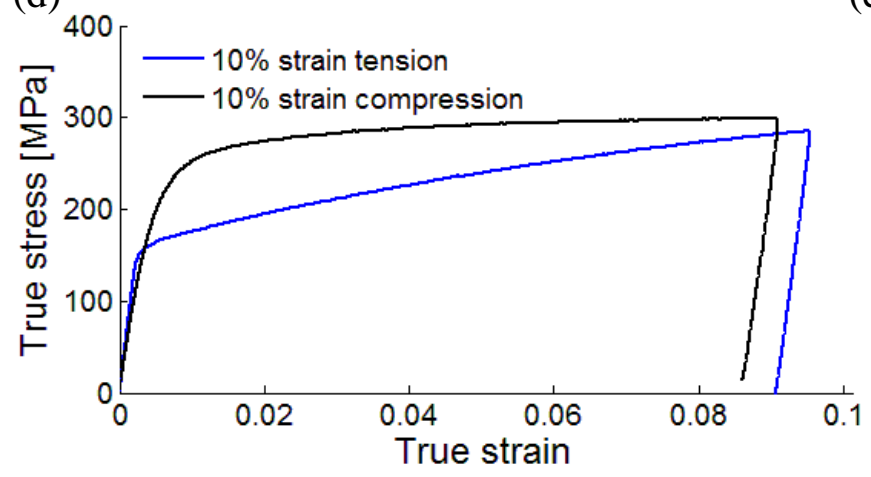

(e)

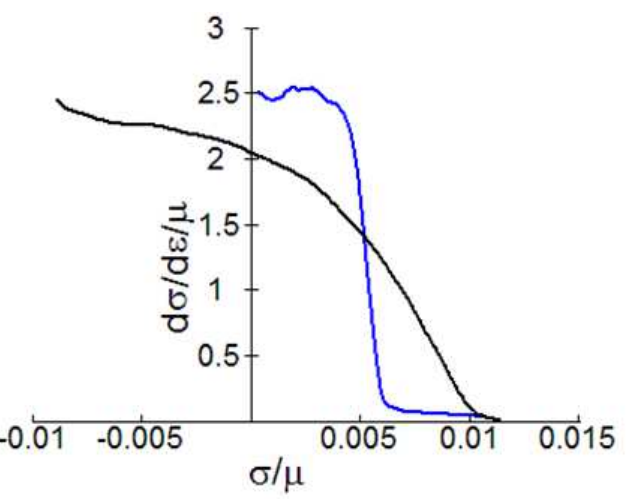

Fig. 2. True stress-true strain response under cyclic tension-compression measured along (a) $\mathrm{RD}$ and (b) TD. Macro-yield points at approximately 0.001 offset are indicated. (c) True stress-true strain curves as a function of accumulated true strain showing drops in yield stress upon load reversal and permanent softening during subsequent straining. (d) True stress-true strain cyclic curve to a strain of $10 \%$ and (e) the corresponding normalized strain hardening rate plots.

\section{Model description}

The EPSC polycrystal model is used for the implementation of the thermally activated dislocation density hardening model featuring forward and reverse motion of dislocations and the slip system kinematic hardening effects. In EPSC, a polycrystal is represented by a set of grains (each having a crystal orientation, an ellipsoidal shape and a volume fraction). Each grain is treated as an elasto-plastic inclusion embedded in a homogeneous effective medium. The effective medium response corresponds to that of the polycrystal and is to be compared with that of the tested sample. Detailed description of the EPSC model can be found in (Hutchinson, 1970; Neil et al.; Turner and Tomé, 1994). Here we present the main equations for completeness. In the following description, we use "." to represent a contracted or dot product and “ $\otimes$ ” for uncontracted or tensor dyadic product.

A linearized relation between homogenized macroscopic stress and strain increments is:

$$
d \boldsymbol{\sigma}=\mathbf{L} d \boldsymbol{\varepsilon} .
$$

where $\mathbf{L}$ is the instantaneous elasto-plastic stiffness tensor of the polycrystal matrix. $\mathbf{L}$ is unknown a priori and is calculated iteratively using a standard self-consistent procedure (Turner and Tomé, 1994). The response of each grain follows from solving the stress equilibrium and compatibility relations for an inclusion embedded in a homogeneous anisotropic matrix under applied loads (Eshelby, 1957; Turner and Tomé, 1994). The macroscopic and the grain scale stress rate and strain rate are related through the following interaction equation:

$$
d \boldsymbol{\sigma}^{c}-d \boldsymbol{\sigma}=-\mathbf{L}^{c^{*}} \cdot\left(d \boldsymbol{\varepsilon}^{c}-d \boldsymbol{\varepsilon}\right)
$$

where $\mathbf{L}^{c^{*}}$ is the effective stiffness given by $\mathbf{L}^{c^{*}}=\mathbf{L}\left(\mathbf{S}^{c^{-1}}-\mathbf{I}\right) . \mathbf{S}^{c}$ is the symmetric Eshelby tensor and $\mathbf{I}$ is the four rank identity matrix. The superscript " $c$ " denotes that a tensor refers to 
the grain scale physical quantity. Using the above constitutive equations, a localization equation for the strain increment can be derived as:

$$
d \boldsymbol{\varepsilon}^{c}=\mathbf{A}^{c} d \boldsymbol{\varepsilon},
$$

where $\mathbf{A}^{c}=\left(\mathbf{L}^{c}+\mathbf{L}^{c^{*}}\right)^{-1}\left(\mathbf{L}^{c^{*}}+\mathbf{L}\right) . \mathbf{L}^{c}$ is the crystal instantaneous elasto-plastic stiffness tensor defined later. Increments in polycrystal stress and strain are equal to the volume average of the grain stress increment and strain increments as:

$$
d \boldsymbol{\sigma}=\left\langle d \boldsymbol{\sigma}^{c}\right\rangle \quad \text { and } \quad d \boldsymbol{\varepsilon}=\left\langle d \boldsymbol{\varepsilon}^{c}\right\rangle,
$$

leading to the following expression: $\mathbf{L}=\left\langle\mathbf{L}^{c} \mathbf{A}^{c}\right\rangle\left\langle\mathbf{A}^{c}\right\rangle^{-1}$. The stress and slip resistances (through the hardening law described later) are updated incrementally for the current deformation step $n+1$, with respect to their previously converged values, noted by the superscript $n$. For example, the new stress of the polycrystalline aggregate is calculated from the overall stress increment, $d \boldsymbol{\sigma}$, associated with the strain increment, $d \boldsymbol{\varepsilon}$, using $\boldsymbol{\sigma}^{n+1}=\boldsymbol{\sigma}^{n}+d \boldsymbol{\sigma}$.

Following the formulation presented in (Wollmershauser et al., 2012), the driving force (resolved shear stress) for the activation of a slip system is not only influenced by the applied loading (type 1 stresses) and inter-granular stresses (type 2 stresses) originating from the EPSC description of grain interactions, $\mathbf{m}^{s} \cdot \boldsymbol{\sigma}^{c}$, but also by the slip system backstress, $\tau_{b s}^{s}$. $\mathbf{m}^{s}$ is the Schmid tensor associated with every slip system $s$. The Schmid tensor is the unit tensor, defined as the symmetric portion of the dyadic product of two orthogonal unit vectors denoting the slip shear direction and the slip plane normal, respectively. In order for a slip system to be active, it must satisfy the following two conditions: (1) $\mathbf{m}^{s} \cdot \boldsymbol{\sigma}^{c}-\tau_{b s}^{s}=\tau_{c}^{s}$, meaning that the resolved shear stress on the $s^{\text {th }}$ slip system has to be equal to the slip resistance reduced for a value of backstress $\left(\tau_{b s}^{s}\right)$ on slip system (stress needs to be on the single crystal yield surface) and (2) $\mathbf{m}^{s} \cdot d \boldsymbol{\sigma}^{c}-d \tau_{b s}^{s}=d \tau_{c}^{s}$, meaning that the $s^{\text {th }}$ slip system has to remain on the evolving single crystal yield surface in a given increment as stress evolves due to hardening. In addition, the formulation allows for only positive shear on a given slip system, i.e., $\Delta \gamma^{s}>0$, since reverse shear of this slip system would be captured as positive slip on the opposite-signed slip system. The slip systems are defined such that the two slip systems sharing the same plane normal, but having opposite sense of shear, are independently considered.

The slip resistance and backstress increments are coupled with the shearing increment of the slip systems through the hardening matrices, $h^{s s^{\prime}}$ and the backstress matrix, $h_{b s}^{s s^{\prime}}$, respectively:

$$
\begin{aligned}
& d \tau_{c}^{s}=\sum_{s^{\prime}} h^{s s^{\prime}} d \gamma^{s^{\prime}} \\
& d \tau_{b s}^{s}=\sum_{s^{\prime}} h_{b s}^{s s^{\prime}} d \gamma^{s^{\prime}}
\end{aligned}
$$


As will be highlighted in the next section, the hardening matrix, $h^{s s^{\prime}}$, follows from the hardening law, while the backstress matrix, $h_{b s}^{s s^{\prime}}$, follows from the evolution of backstress equations with shear strain.

For the individual grains, the constitutive relation between stress increment $\left(d \boldsymbol{\sigma}^{c}\right)$ and increment in total strain $\left(d \boldsymbol{\varepsilon}^{c}\right)$ is:

$$
d \boldsymbol{\sigma}^{c}=\mathbf{C}^{c} \cdot\left(d \boldsymbol{\varepsilon}^{c}-\sum_{s} \mathbf{m}^{s} d \gamma^{s}\right)
$$

where $\mathbf{C}^{c}$ is the single crystal elastic stiffness tensor and $\sum_{s} \mathbf{m}^{s} d \gamma^{s}$ is the plastic strain increment calculated as the sum of shear increments, $d \gamma^{s}$, over all slip systems per grain. Only slip systems that are active will have non-zero shear increment, $d \gamma^{s}$. Similar to the macroscopic stress and strain increments, a linear relationship between grain stress and total strain increment can be written as:

$$
d \boldsymbol{\sigma}^{c}=\mathbf{L}^{c} d \boldsymbol{\varepsilon}^{c} .
$$

From Eqs. (5, 7, and 8), $\mathbf{L}^{c}$ becomes:

$$
\mathbf{L}^{c}=\mathbf{C}^{c} \cdot\left(\mathbf{I}-\sum_{s} \mathbf{m}^{s} \otimes \mathbf{f}^{s}\right)
$$

where:

$$
\mathbf{f}^{s}=\sum_{s^{\prime}}\left(\mathrm{X}^{-1}\right)^{s s^{\prime}} \mathbf{m}^{s^{\prime}} \cdot \mathbf{C}^{c}
$$

The matrix, $\mathbf{f}^{s}$, relates an increment in shear strain on individual slip system with the total strain increment in crystal:

$$
d \gamma^{s}=\mathbf{f}^{s} \cdot d \mathbf{\varepsilon}^{c}
$$

Since the activation condition includes the backstress term, the definition of the $X^{s s^{\prime}}$ matrix takes the following form:

$$
X^{s s^{\prime}}=h^{s s^{\prime}}+h_{b s}^{s s^{\prime}}+\mathbf{m}^{s} \cdot \mathbf{C}^{c} \cdot \mathbf{m}^{s^{\prime}}
$$

For completeness, we briefly reflect on the crystallographic texture evolution calculations in EPSC. In the model, the plastic rotation rate, $\mathbf{W}^{p}$, of each crystal is related to the shear rates on the individual slip systems via the following kinematic formula:

$$
\mathbf{W}^{p}=\sum_{s} \dot{\gamma}^{s} \mathbf{q}^{s}
$$

The tensor $\mathbf{q}^{s}$ is the unit slip system tensor, defined as the skew symmetric portion of the dyadic product of two orthogonal unit vectors denoting the slip shear direction and the slip plane normal, respectively. The lattice rotation rate, $\mathbf{W}^{*}$, is calculated as:

$$
\mathbf{W}^{*}=\mathbf{W}^{a p p}+\boldsymbol{\Pi}-\mathbf{W}^{p},
$$

where $\mathbf{W}^{a p p}$ is the macroscopically applied rotation rate of the grain shape and $\boldsymbol{\Pi}$ is the reorientation contribution from the antisymmetric part of the Eshelby tensor (Lebensohn and Tomé, 1993). The tensor $\mathbf{W}^{*}$ is used to update the crystal orientation and hence to calculate texture evolution. 


\subsection{Hardening law based on dislocation density}

In the present model, the resistance to slip, $\tau_{c}^{s}$, evolves with the rate of dislocation storage, which is governed by thermally activated dislocation glide and hence is dependent on strain rate and temperature. In several prior studies, a similar hardening model has been successfully applied within the self-consistent models to a variety of metals of different crystal structures: hexagonal close packed (HCP) Zr (Beyerlein and Tomé, 2008; Knezevic et al., 2013b; Knezevic and Landry, 2015; Knezevic et al., 2014e; Knezevic et al., 2015), Be (Brown et al., 2012; Knezevic et al., 2013a; Zecevic et al., 2015a), Mg (Beyerlein et al., 2011; Lentz et al., 2015a; Lentz et al., 2015b), body centered cubic (BCC) Ta (Ardeljan et al., 2014a; Ardeljan et al., 2014b; Bhattacharyya et al., 2015; Knezevic et al., 2014a; Knezevic et al., 2014e), a face centered cubic (FCC) cobalt alloy (Knezevic et al., 2014b), and orthorhombic uranium (Knezevic et al., 2012; Knezevic et al., 2013d; Knezevic et al., 2013e). However, to meet the objectives of this work, we found it necessary to extend the model to include the kinematic hardening at the slip system level and reverse dislocation motion accompanied with the dynamic recovery. Thus, the basic formulation of the hardening model is briefly reviewed.

In the following, the Greek superscript $\alpha$ represents the $\{111\}\langle 110\rangle$ slip family for FCC metals and the superscripts $s, s$ ' span over the individual slip systems belonging to the family along their arbitrarily chosen positive $s^{+}$and negative $s^{-}$directions. The resistance to slip is defined as:

$$
\tau_{c}^{s}=\tau_{o, f}^{\alpha}+\tau_{o, H P}^{\alpha}+\tau_{f o r}^{s}+\tau_{d e b}^{\alpha}
$$

where $\tau_{o, f}^{\alpha}$ is a friction stress (dependent on the Peierls stress and the initial content of dislocations), $\tau_{o, H P}^{\alpha}$ is the Hall-Petch-like term dependent on the initial grain size, $\tau_{f o r}^{s}$ is the forest term dependent on statistically stored dislocations, and $\tau_{d e b}^{\alpha}$ is the debris term dependent on amount of dislocations stored as ordered defects.

Effect of grain size on initial slip resistance is modeled using a Hall-Petch-like relation:

$$
\tau_{o, H P}^{\alpha}=\mu H P \sqrt{\frac{b^{\alpha}}{d_{g}}}
$$

where $\mu$ is the shear modulus, $H P$ is a Hall-Petch coefficient and $d_{g}$ is the initial grain size. Note that $\tau_{o, f}^{\alpha}$ and $\tau_{o, H P}^{\alpha}$ define the initial slip resistance $\tau_{o}^{\alpha}=\tau_{o, f}^{\alpha}+\tau_{o, H P}^{\alpha}$ and do not evolve with strain. In the present study the $\tau_{o, H P}^{\alpha}$ term was not characterized separately but included in the initial slip resistance term, $\tau_{o}^{\alpha}$.

Contribution of forest dislocation population to the slip resistance is given by the Taylor relation (Beyerlein and Tomé, 2008; Mecking and Kocks, 1981):

$$
\tau_{\text {for }}^{s}=b^{\alpha} \chi \mu \sqrt{\rho_{\text {tot }}^{s}}
$$


where $b^{\alpha}=2.86 \times 10^{-10} \mathrm{~m}$ is the Burgers vector of the slip mode, $\chi$ is the dislocation interaction constant taken to be 0.9 (Lavrentev, 1980), and $\rho_{t o t}^{s}$ is the total forest dislocation density for the $s^{\text {th }}$ slip system $(s \in \alpha)$.

Contribution of the dislocation substructures (debris) to the slip resistance is defined through the following relation (Madec et al., 2003):

$$
\tau_{d e b}^{\alpha}=k_{d e b} \mu b^{\alpha} \sqrt{\rho_{d e b}} \log \left(\frac{1}{b^{\alpha} \sqrt{\rho_{d e b}}}\right),
$$

where $k_{d e b}=0.086$ is a material independent constant and $\rho_{d e b}$ is the density of dislocations stored as substructure. Justification for the Eq. (18) arises from the basic thermally activated processes such as climb and cross slip, which are responsible for pattern formation (Jackson, 1985; Kocks and Mecking, 2003; Peeters et al., 2001a; Wang et al., 2007). Dislocation in these patters self-organize into lower energy patterns, such as cells and cell walls (e.g. Kuhlmann-Wilsdorf, 1989). While the hardening model considers these processes to contribute recovery of forest dislocations, it also assumes that a smaller fraction of the recovered forest dislocations contribute to substructure development. The model, however, neglects the length scales associated with substructure. As our present interests lie in modeling cyclic deformation where dislocation cell walls processes take place, advancing the substructure model is left for future work. Evolution of the dislocation densities, $\rho_{t o t}^{s}$ and $\rho_{\text {deb }}$, is described next.

As explained earlier, accurate modeling of the strain-path changes deformation must account not only for the microstructure induced anisotropy during pre-loading, but also for instantaneous rearranging of the microstructure upon reloading in order to predict the change in the initial yield stress and subsequent hardening. An essential element of the developed model is the introduction of reversible dislocations defined as a fraction of the previously stored dislocations that progressively annihilate upon strain reversal. These dislocations, since loosely tangled, re-emit from pile-ups and substructures with very low slip resistance, influencing the hardening behavior upon reloading. An important feature of the model is that it is able to detect the local slip system shear reversal in each grain, which is important since even if the macroscopic strain is reversed, locally each slip system in each grain may not experience an exact reverse shear.

The total dislocation density is directionally broken into a forward and a reverse on the individual slip systems per grain and explicitly related to the shear activity in each slip system. The approach was implemented within VPSC and successfully applied to capturing the behavior of low carbon steels during strain path changes (Kitayama et al., 2013). In particular, the plateau with low hardening rate present during reloading after large pre-strains was successfully captured.

Several previous studies have shown that during forward deformation of AA6022-T4 dislocations can organize in cell walls, while during loading in the reverse direction existing cell walls dissolve and new cells walls form (Christodoulou et al., 1986; Hasegawa et al., 1975b; Trivedi et al., 2004). These microstructural processes are expected to influence the BE 
and subsequent work hardening. However, a study on strain reversal performed on mild steels (Rauch, 1997) found weak to no relationship between the organization of dislocations in cell walls and the mechanical behavior upon strain reversal. These findings were further exploited and reinforced in a combined modeling and experimental study (Rauch et al., 2007), where a reversible dislocation law without an explicit treatment of cell walls was able to predict the $\mathrm{BE}$ and work hardening upon strain reversals of several alloys including AA6022-T4, AA1050-O and a low carbon steel.

The directionality in the dislocation based hardening law is introduced as follows. As mentioned earlier each slip system $s$ is split into two systems denoted as $s^{+}$and $s^{-}$having the same slip plane but opposite slip direction: $\mathbf{b}$ and $-\mathbf{b}$. From this point onwards, we use superscript $s$ to denote variables that are equal for both slip directions, while $s^{+}$and $s^{-}$to label variables related to a particular slip direction. The total dislocation density on each slip system consists of:

$$
\rho_{\text {tot }}^{s}=\rho_{\text {for }}^{s}+\rho_{r e v}^{s^{+}}+\rho_{r e v}^{s^{-}},
$$

where $\rho_{\text {for }}^{s}$ is the forward dislocation density common to both directions $s^{+}$and $s^{-}$and $\rho_{r e v}^{s^{+}}$ and $\rho_{\text {rev }}^{s-}$ are the reversible dislocation density populations associated with $s^{+}$and $s^{-}$ directions, respectively.

The dislocation density populations follow laws that depend on previous strain history and shearing direction on slip systems. For the forward dislocation density population, the evolution law is based on the thermal activation (Kocks and Mecking, 1981):

$$
\frac{\partial \rho_{f o r}^{s}}{\partial \gamma^{s}}=(1-p) \frac{\partial \rho_{g e n, f o r}^{s}}{\partial \gamma^{s}}-\frac{\partial \rho_{r e m, f o r}^{s}}{\partial \gamma^{s}}=(1-p) k_{1}^{\alpha} \sqrt{\rho_{t o t}^{s}}-k_{2}^{\alpha}(\dot{\varepsilon}, T) \rho_{f o r}^{s}, d \rho_{f o r}^{s}=\frac{\partial \rho_{f o r}^{s}}{\partial \gamma^{s}}\left|d \gamma^{s}\right|,
$$

where $k_{1}^{\alpha}$ is an adjustable coefficient accounting for rate of generation of statistically stored dislocations, $k_{2}^{\alpha}$ is a rate-sensitive coefficient capturing dynamic recovery (Beyerlein and Tomé, 2008) and $p$ is a reversibility parameter. The latter coefficient, $k_{2}^{\alpha}$, is given by:

$$
\frac{k_{2}^{\alpha}}{k_{1}^{\alpha}}=\frac{\chi b^{\alpha}}{g^{\alpha}}\left(1-\frac{k T}{D^{\alpha} b^{3}} \ln \left(\frac{\dot{\varepsilon}}{\dot{\varepsilon}_{o}}\right)\right),
$$

where, $k, \dot{\varepsilon}_{o}, g^{\alpha}$, and $D^{\alpha}$ are the Boltzmann constant, a reference strain rate taken to be $10^{7}$, an effective activation enthalpy and a drag stress, respectively. Dynamic recovery is often associated with thermal activation of dislocation cross-slip and climb, and the formation of dislocation debris is concomitant with these recovery processes. As a consequence, in the model, the rate of debris development is coupled to the rate of recovery of all active dislocations through:

$$
d \rho_{d e b}=\sum_{s} q^{\alpha} b^{\alpha} \sqrt{\rho_{d e b}} \frac{\partial \rho_{r e m, f o r}^{s}}{\partial \gamma^{s^{\prime}}}\left|d \gamma^{s^{\prime}}\right|
$$

where $q^{\alpha}$ is a dislocation recovery rate coefficient defining the fraction of $\alpha$-type dislocations that do not annihilate but become debris. 
The reversibility parameter divides increments in the total dislocation density $\left(k_{1}^{\alpha} \sqrt{\rho_{t o t}^{s}} d \gamma^{s}\right)$ into: the forward $\left((1-p) k_{1}^{\alpha} \sqrt{\rho_{t o t}^{s}} d \gamma^{s}\right)$ and the reversible $\left(p k_{1}^{\alpha} \sqrt{\rho_{\text {tot }}^{s}} d \gamma^{s}\right)$. The value of the reversibility parameter is taken to be unity, meaning that all statistically stored dislocation densities are reversible, which is suggested in (Kitayama et al., 2013) to be a good approximation up to pre-strains of 0.3 . While the evolution of the forward dislocation population, $\rho_{\text {for }}^{s}$, is independent on the shearing direction on a slip system, the reversible dislocations populations evolve with a particular direction of shearing. If the direction of shearing is positive, $d \gamma^{s+}>0$, then the law is as follows:

$$
\begin{aligned}
& \frac{\partial \rho_{r e v}^{s^{+}}}{\partial \gamma^{s}}=p k_{1}^{\alpha} \sqrt{\rho_{t o t}^{s}}-k_{2}^{\alpha}(\dot{\varepsilon}, T) \rho_{r e v}^{s^{+}}, \\
& \frac{\partial \rho_{r e v}^{s^{-}}}{\partial \gamma^{s}}=-k_{1}^{\alpha} \sqrt{\rho_{t o t}^{s}}\left(\frac{\rho_{r e v}^{s^{-}}}{\rho_{0}^{s}}\right)^{m},
\end{aligned}
$$

where $m$ is the parameter taken to be 0.5 controlling rate of dislocation recombination (Wen et al.) and $\rho_{0}^{s}$ is the total density that was present when the shear was reversed on the $s^{\text {th }}$ slip system (Kitayama et al., 2013). The relation for decay of reversible dislocation densities with shear strain, 23b, was proposed and physically justified in (Kitayama et al., 2013; Rauch et al., 2007), but without parameter $m$, which was added in (Wen et al.) to increase rate of dislocation recombination at the start of reversal and decrease the rate towards the end of reversal. The underlying physical justification for Eq. (23b) is that the rate of recombination of dislocations moving backward is limited by the storage rate they had when moving forward and that the process of recombination decreases and vanishes with $\rho_{\text {rev }}^{s^{-}}$. If shearing occurs in the negative direction, $d \gamma^{s-}>0$, the increment assigned to $\rho_{\text {rev }}^{s^{-}}$evolution is analogous to that presented in (23a), while the increment assigned to $\rho_{r e v}^{s^{+}}$evolution is equal to that of (23b). The initial conditions for the dislocation population evolution laws are: $\rho_{\text {for }}^{s}\left(\gamma^{s}=0\right)=\rho_{\text {initial }}^{s}, \quad \rho_{\text {rev }}^{s^{+}}\left(\gamma^{s}=0\right)=0$ and $\rho_{\text {rev }}^{s^{-}}\left(\gamma^{s}=0\right)=0$. The initial dislocation density, $\rho_{\text {initial }}^{s}$, was initialized to a small value of $10^{12} \mathrm{~m}^{-2}$.

Based on Eq. (5), the evolution of the resistance on a slip system is done via instantaneous hardening coefficients $h^{s s^{\prime}}$. Current slip resistance, $\tau_{c}^{s}$, on a slip system $s$ is a function of dislocation densities $\left(\rho_{\text {for }}^{s}\right.$ and $\left.\rho_{d e b}\right)$, which are in turn the function of the shearing strain on slip systems inside crystal $\left(\gamma^{s}\right)$. This implies that we must evaluate:

$$
h^{s s^{\prime}}=\frac{\partial \tau_{c}^{s}}{\partial \gamma^{s^{\prime}}},
$$

which can be further expanded to:

$$
\frac{\partial \tau_{c}^{s}}{\partial \gamma^{s^{\prime}}}=\frac{\partial \tau_{o}^{\alpha}}{\partial \gamma^{s^{\prime}}}+\frac{\partial \tau_{f o r}^{s}}{\partial \gamma^{s^{\prime}}}+\frac{\partial \tau_{d e b}^{\alpha}}{\partial \gamma^{s^{\prime}}}+\frac{\partial \tau_{o, H P}^{\alpha}}{\partial \gamma^{s^{\prime}}}
$$


Using the chain rule and based on the above equations, individual terms from equation (25) evaluate to:

$$
\begin{aligned}
& \frac{\partial \tau_{o}^{\alpha}}{\partial \gamma^{s^{\prime}}}=\frac{\partial \tau_{o, H P}^{\alpha}}{\partial \gamma^{s^{\prime}}}=0 \\
& \frac{\partial \tau_{f o r}^{s}}{\partial \gamma^{s^{\prime}}}=\frac{\partial \tau_{f o r}^{s}}{\partial \rho_{t o t}^{s^{\prime}}} \frac{\partial \rho_{t o t}^{s^{\prime}}}{\partial \gamma^{s^{\prime}}}=b^{\alpha} \chi \mu \frac{1}{2 \sqrt{\rho_{t o t}^{s}}}\left(\frac{\partial \rho_{f o r}^{s^{\prime}}}{\partial \gamma^{s^{\prime}}}+\frac{\partial \rho_{r e v}^{s^{\prime}+}}{\partial \gamma^{s^{\prime}}}+\frac{\partial \rho_{r e v}^{s^{\prime}-}}{\partial \gamma^{s^{\prime}}}\right), \text { if } s=s^{\prime} \text { else } \frac{\partial \tau_{f o r}^{s}}{\partial \gamma^{s^{\prime}}}=0 \\
& \frac{\partial \tau_{d e b}^{\alpha}}{\partial \gamma^{s^{\prime}}}=\frac{\partial \tau_{d e b}^{\alpha}}{\partial \rho_{d e b}} \frac{\partial \rho_{d e b}}{\partial \gamma^{s^{\prime}}}=-k_{d e b} \mu b^{\alpha}\left[\log \left(b^{\alpha} \sqrt{\rho_{d e b}}\right)+1\right] \frac{1}{2 \sqrt{\rho_{d e b}}} \frac{\partial \rho_{d e b}}{\partial \gamma^{s^{\prime}}}
\end{aligned}
$$

\subsection{Evolution law for backstresses}

As mentioned earlier, the introduction of kinematic hardening is necessary for capturing the micro plasticity processes responsible for non-linear unloading and the BE. In order for crystals to start micro-yielding, the following condition has to be fulfilled: $\mathbf{m}^{s} \cdot \boldsymbol{\sigma}^{c}-\tau_{b s}^{s}=\tau_{c}^{s}$. Satisfying this condition is controlled in part by evolution of the backstresses. Here, we extend the EPSC model to account for the effect of intra-granular micro backstresses at the slip system level.

Several evolution laws for backstress with shear strain have been proposed in the literature. The Armstrong and Frederick model (Armstrong and Frederick, 1966) has been adopted in crystal plasticity and successfully employed in several prior studies (Li et al., 2014; Li et al., 2012; Xu and Jiang, 2004). Another phenomenological law for the evolution of backstress based on a modified Voce hardening law was used to simulate the cyclic deformation of stainless steel (Wollmershauser et al., 2012). However, these studies dealt with small prestrains ( 0.02) and multiple cycles. A model for the evolution of backstress based on experimental observations (Wilson and Bate, 1986) applicable to large pre-strains has been presented in (Beyerlein and Tomé, 2007). The model was successfully applied in VPSC to pre-strains of $0.08,0.18$ and 0.28 followed by reloading. We adapt this model for the evolution of backstress in the present study dealing with large pre-strains in EPSC.

The origin of backstresses in $\mathrm{Al}$ alloys is the stress field arising from dislocation loops formed around precipitates (Proudhon et al., 2007). When a dislocation surpasses a nonshearable obstacle such as a precipitate particle present in Al alloys (Miao and Laughlin, 1999; Trivedi et al., 2004), it leaves a dislocation loop around it. During plastic deformation, dislocation loops keep accumulating around the precepitates and the backstress field strengthens (Hart, 1972; Proudhon et al., 2007). Thus evolution of the backstresses is directly proportional to the number of dislocation loops formed around precipitates. Analogous expression for the evolution of the number of dislocation loops to the law adopted here from (Beyerlein and Tomé, 2007) for the evolution of backstresses with shear strain has been suggested in (Proudhon et al., 2007), which justifies the use of the backstress law for precipitation hardened alloys. 
In what follows, the evolution laws for the slip system backstresses are described during the course of forward loading (pre-strain) and unloading followed by reverse loading. Schematic illustration defining direct and opposite slip systems $s^{+}$and $s^{-}$under the micro backstresses is shown in Fig. 3.

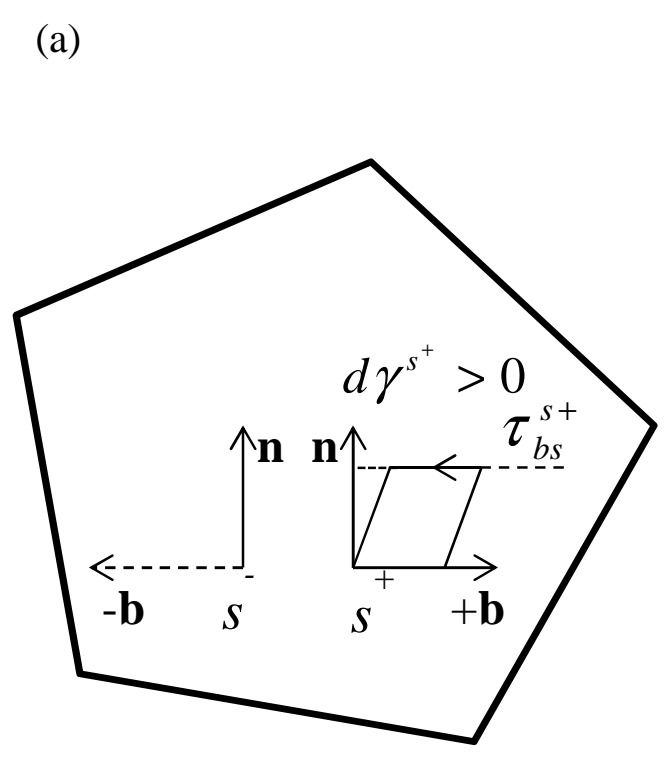

(b) FORWARD LOADING

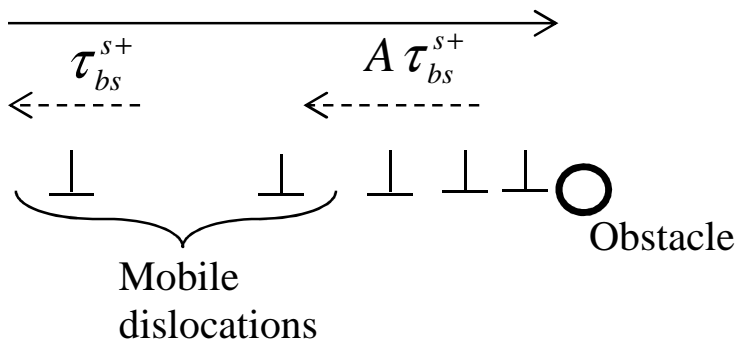

(c) UNLOADING

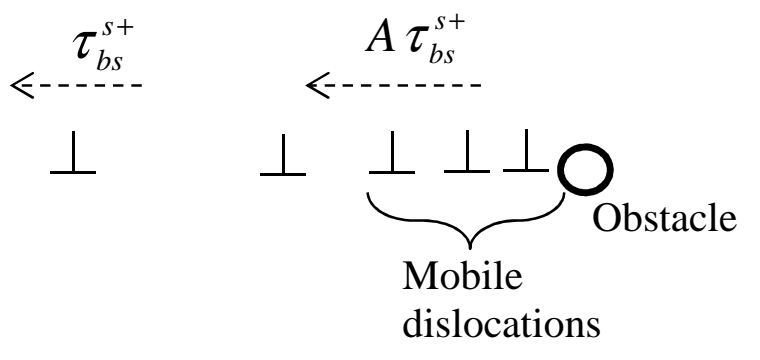

Fig. 3. Schematics showing (a) a given slip system $s$ split into $s^{+}$and $s^{-}$under action of the directional micro backstresses formed during slip in the $+\mathbf{b}$ direction $\left(d \gamma^{s+}>0\right)$, (b) the long range micro backstresses acting on the dislocations that are primary mobile during forward loading and (c) the near pile-up micro backstresses acting on the dislocations that are primary mobile during unloading.

\subsubsection{Evolution of backstresses during forward loading}

Similarly to the directional definition of reversible dislocation, the backstresses associated with each slip system are defined directionally. We recall that each slip system is divided into two systems $s^{+}$and $s^{-}$having the same slip plane normal $\mathbf{n}$ but opposite slip directions: $\mathbf{b}$ and -b which is illustrated in Fig. 3a. If the direction of shearing is positive, $d \gamma^{s+}>0$, then the evolution of backstresses is governed by the following law:

$$
\begin{aligned}
& \tau_{b s}^{s+}=\tau_{b s}^{s a t}\left(1-\exp \left(-v \gamma^{s+}\right)\right), \\
& \tau_{b s}^{s-}=-A \tau_{b s}^{s+}
\end{aligned}
$$

where $\tau_{b s}^{s+}$ and $\tau_{b s}^{s-}$ are backstresses in the two opposite directions per slip system, $\tau_{b s}^{s a t}$ is a saturation value for backstresses, A and $v$ are adjustable material parameters and $\gamma^{s+}$ is the accumulated value of shear strain on that slip system.

The slip system backstress, $\tau_{b s}^{s+}$, acts in the direction which is opposite to the resolved shear stress on that slip system (Fig. $3 b$ ). Therefore, $\tau_{b s}^{s+}$ lowers the activation stress i.e.: 
$\mathbf{m}^{s+} \cdot \boldsymbol{\sigma}^{c}-\tau_{b s}^{s+}=\tau_{c}^{s+}$. At the same time, $\tau_{b s}^{s+}$ acts in the direction of resolved shear stress on the slip system $s^{-}$meaning that the backstress on the $s^{-}$system is of the opposite sign i.e. $\tau_{b s}^{s-}=-\tau_{b s}^{s+}$. Therefore, $\tau_{b s}^{s-}$ aids the resolved shear stress according to: $\mathbf{m}^{s-} \cdot \boldsymbol{\sigma}^{c}-\tau_{b s}^{s-}=\tau_{c}^{s-}$. However, the backstress acting on the slip system $s^{-}$is much higher than on the slip system $s^{+}$. We now turn our attention to a physical justification of the scaling parameter A in Eq. $27 b$.

During forward loading majority of plastic deformation is accommodated by the dislocations that are under long-range internal stresses, while a much smaller portion of plastic strain is accommodated by decreasing spacings between the dislocations in a pile-up (Fig. 3b). Upon local unloading, the dislocations in a pile-up mobilize because stress acting on them in the forward direction decreases (Fig. 3c). With the unloading process, dislocation pile-ups expand accommodating some micro shear strain in the reverse direction. Driving force for these processes are believed to be the micro backstresses of high magnitude arising from pileups (Sritharan and Chandel, 1997). We relate these micro backstresses to the long range backstresses by parameter A. Dislocation pile-ups also contribute to the long range stresses (Kassner et al., 2013; Kim et al., 2012; Seeger, 1957), therefore relating the pile-up micro backstresses to the long range backstresses is plausible. Dislocations re-emitted from pile-ups are believed to experience significantly lower resistance to their motion in the backward direction (Sritharan and Chandel, 1997). Therefore, justification for a large value of the parameter A is twofold: (1) the large micro backstress arising from pile-ups and (2) the low resistance to slip of dislocations re-emitted from pile ups. The predicted value for A, based on our model, is $\sim 10$.

\subsubsection{Evolution of backstresses upon unloading followed by reverse loading}

As mentioned earlier, the physical origin of nonlinear unloading is in the short range motion of dislocations re-emitted from pile-ups under action of the micro backstresses that have a large magnitude (Cleveland and Ghosh, 2002; Sritharan and Chandel, 1997). During unloading these micro backstresses relax while pile-ups expand giving rise to a micro plastic strains in the reverse direction $\left(d \gamma^{s-}>0\right)$. The slip system $s^{-}$can continues to be active after the local micro backstresses relax due to action of the applied stress in the reverse direction (type 1 stress). To this end, an evolution law for $\tau_{b s}^{s-}$ must capture: (1) a rapid nullification followed by (2) a saturation type evolution law in the reverse direction. The saturation value for $\tau_{b s}^{s-}$ is conveniently chosen to be the backstress at the point of strain reversal (Beyerlein and Tomé, 2007; Wilson and Bate, 1986; Wilson et al., 1990). An appropriate evolution law for $\tau_{b s}^{s-}$ during unloading followed by loading in the reversed direction, which fulfills the above defined conditions, is expresses as:

$$
\tau_{b s}^{s-}=-(A+1) \tau_{b s 0}^{s+} \exp \left(-\frac{\gamma^{s-}}{\gamma_{b}}\right)+\tau_{b s 0}^{s+}
$$




$$
\tau_{b s}^{s+}=-A \tau_{b s}^{s-}
$$

where $\tau_{b s 0}^{s+}$ is the value of backstress at the point of the strain reversal and $\gamma_{b}$ is another adjustable material parameter. Eq. (28a) evolves $\tau_{b s}^{s-}$ with accumulated shear strain $\gamma^{s-}$, from an initial value of $-A \tau_{b s 0}^{s+}$ to a new saturation value conveniently chosen to be the backstress at the point of reversal. Note that $s^{+}$is now inactive and that $\tau_{b s}^{s+}$ and $\tau_{b s}^{s-}$ are related using the same parameter A as used in Eq. 27.

Based on Eq. (6), the evolution of the backstress on a slip system is done via instantaneous hardening coefficients $h_{b s}^{s s^{\prime}}$ defined as:

$$
\begin{aligned}
& h_{b s}^{s s}=\frac{d \tau_{b s}^{s}}{d \gamma^{s}}=\tau_{b s}^{s a t} \nu \exp \left(-v \gamma^{s}\right) \\
& h_{b s}^{s s}=\frac{d \tau_{b s}^{s}}{d \gamma^{s}}=\frac{(A+1) \tau_{b s 0}^{s} \exp \left(-\frac{\gamma^{s}}{\gamma_{b}}\right)}{\gamma_{b}}
\end{aligned}
$$

where $s$ span over the available slip systems in positive, $s^{+}$, and negative, $s^{-}$, directions. The off diagonal terms evaluate to zero.

In the next section, this model is used to predict the cyclic stress-strain response of the polycrystalline AA6022-T4 alloy. We calibrate and critically validate the model against the experimental data presented in section 2 and provide insights into the effect of the residual stresses, backstresses, and reversible dislocations on various aspects of the elasto-plastic material response during cyclic loading to large pre-strains.

\section{Results and discussion}

We represent the initial texture (Fig. 1b) of the material using 1000 weighted orientations and assign an initially spherical shape to the representative ellipsoids. Loading in cyclic tension and compression was simulated by imposing strain increments along RD or TD, while enforcing zero average stress along the lateral two sample directions. As deformation proceeds, grains are allowed to change their crystal orientation and shape to become ellipsoids.

\subsection{Calibration of parameters}

We compared simulated and measured $10 \%$ tensile true stress-true strain curves to determine the following slip system material parameters: initial slip resistance, $\tau_{o}^{\alpha}$, trapping rate coefficient, $k_{1}^{\alpha}$, activation barrier for de-pinning, $g^{\alpha}$, and drag stress, $D^{\alpha}$. Each of these parameters affects different portions of the stress-strain curve. For example, $\tau_{o}^{\alpha}$ is responsible for the yield stress and $k_{1}^{\alpha}$ mainly governs the initial slope of the stress-strain curve. Consequently, it is possible to obtain a reasonable estimate of these parameters even from one stress-strain curve. To establish the kinematic backstress parameters, we utilized the entire $10 \%$ cyclic curve. The parameters were only slightly corrected to better fit the RD $2 \%$ cyclic curve. The corresponding set of parameters is given in Tables I and II. The remaining 
experimental cyclic curves were used to test the model's predictive capabilities with this set. The comparison of the model (dash) and experimental curves (solid) is given in Fig. 4.

Table I. Constitutive parameters for evolution of slip resistance.

\begin{tabular}{|l|l|l|l|l|}
\hline$\tau_{0}^{\alpha}[M P a]$ & $k_{1}^{\alpha}\left[m^{-1}\right]$ & $g^{\alpha}$ & $D^{\alpha}[M P a]$ & $q^{\alpha}$ \\
\hline 51 & $2.1 \times 10^{8}$ & 0.02 & 2500 & 16 \\
\hline
\end{tabular}

Table II. Parameters for evolution of kinematic hardening.

\begin{tabular}{|l|l|l|l|}
\hline$\tau_{b s}^{\text {sat }}$ & $\boldsymbol{v}$ & $\gamma_{b}$ & $A$ \\
\hline 10 & 60 & 0.0008 & 10 \\
\hline
\end{tabular}

\subsection{Predictions of mechanical response}

From the comparison between simulated and measured curves, it is evident that the model is capable of predicting all of the particularities associated with cyclic tension-compression deformation of the AA6022-T4 alloy.

We observe that the flow stresses and hardening rates during monotonic tension are well captured. The tensile curve exhibits a classical decreasing hardening rate throughout. Upon strain reversal, the model remarkably captures non-linear unloading as well as the drop in yield stress. Subsequently, there is a more pronounced decrease in hardening rate during compression in the reverse direction due to the annihilation of loosely tangled dislocations. The model captures this shift in the hardening rate. Finally, to explore plastic anisotropy, cyclic tests were simulated in the transverse direction. Figure $4 \mathrm{~b}$ reveals a small plastic anisotropy between RD and TD attributed to the initial crystallographic texture.

A key achievement of this work is that the model is shown to provide very good agreement for all cyclic stress-strain responses using a single set of parameters. Specifically, the small plastic anisotropy, hardening rates, non-linear unloading, BE and permanent softening are simultaneously captured. As many of these features in flow stress of AA6022-T4 are similar to those in other cubic metals, the present modeling framework is expected to be useful for a wider set of materials in addition to $\mathrm{Al}$ alloys. 
(a)

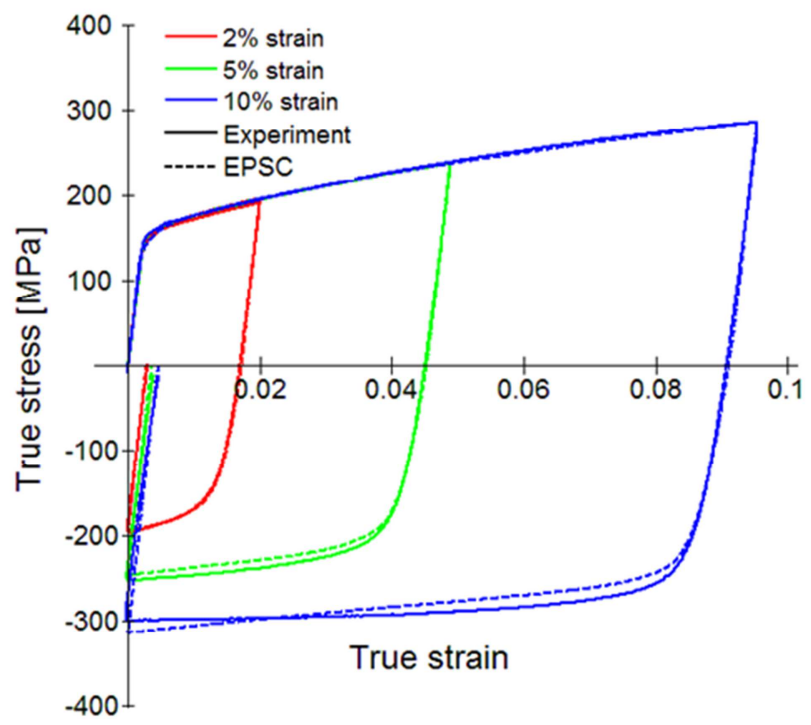

(b)

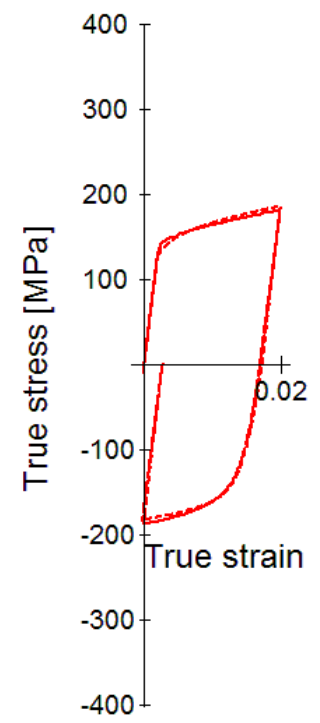

Fig. 4. Measured and simulated true stress-true strain response of AA6022-T4 samples in cyclic tension-compression along (a) RD and (b) TD. The solid lines are the experimental curves and the dashed lines are the model calculations.

Good predictions of the cyclic mechanical behavior were the result of a combination of three distinct mechanisms incorporated in the model. The inter-granular residual stresses, backstresses, and reversible dislocations mechanisms were all active in the simulations. To isolate the individual contribution of these mechanisms on the material response during cyclic loading, we performed the RD simulations without the contributions of (a) the residual stresses, (b) the backstresses, (c) the residual stress and backstresses, and (d) the reversible dislocations. The effects are shown in Fig. 5. 

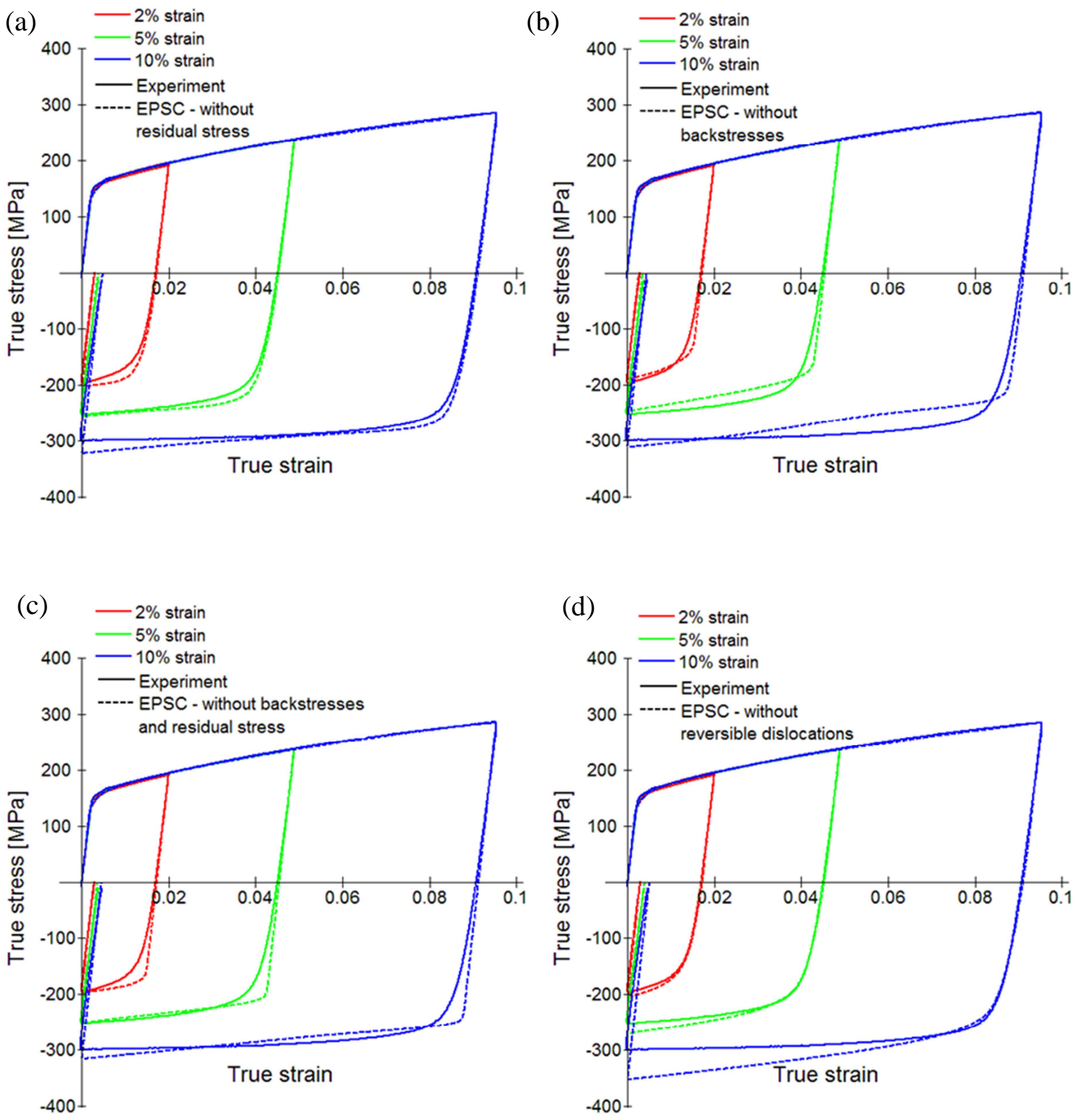

Fig. 5. Measured and simulated true stress-true strain response of AA6022-T4 samples in cyclic tension-compression along RD (a) without residual stresses, (b) without backstresses, (c) without residual and backstresses, and (d) without reversible dislocations. The solid lines are the experimental curves and the dashed lines are the model calculations.

\subsubsection{Effect of residual stress}

Figure 5a shows experimental results together with the EPSC predictions without consideration of the residual stresses upon reloading. From the comparison between Fig. 4 and Fig. 5a, it is evident that inter-granular residual stresses are aiding plastic deformation in the opposite direction (in this case the compression). To more clearly illustrate the effect of the inter-granular residual stress on subsequent loading, we compare the material response after continuous deformation in tension and in compression in Fig. 6. The starting point of the 
continuous deformation is the unloaded state, i.e. zero overall macroscopic stresses (type 1 stresses). Thus, during subsequent loading, only type 1 stresses contribute to the left hand side of the activation condition for the slip systems $\left(\mathbf{m}^{s} \cdot \boldsymbol{\sigma}^{c}=\tau_{c}^{s}\right)$. With inter-granular residual stresses (the type 2 stresses) being erased, the model predicts a decrease in yield stress in tension as well as in compression compared to that at the end of tension (the difference is around $40 \mathrm{MPa}$ ). In this case, both the tensile and the compressive yield stresses are the same (the response is symmetric). This is because the response is controlled by the slip resistance, $\tau_{c}^{s}$, recorded at the end of tension pre-straining. In contrast, when the intergranular stresses are present, the response is asymmetric. While the yield stress in tension returns to the original value upon continued loading, the yield stress in compression drops more. This behavior is due to the inter-granular residual stresses that are "aiding" the deformation in reverse direction. The difference in yield stress in compression vs. tension comes from the left hand side of the activation law reflecting the stress state in grains.

The effect of residual stress on the shift in yield stress in compression is more significant at larger pre-strains. The similar effects of residual stress on the cyclic deformation were also reported in ( $\mathrm{Li}$ et al., 2014), where an analysis of the effect of residual stress was performed at small pre-loads. Additionally, strong effects of the residual stresses on the material response of an AA6111 aluminum alloy after pre-straining have been highlighted in (Wu et al., 2005). Interestingly, residual stresses do not play a major role for the non-linear unloading (Fig. 5a). 


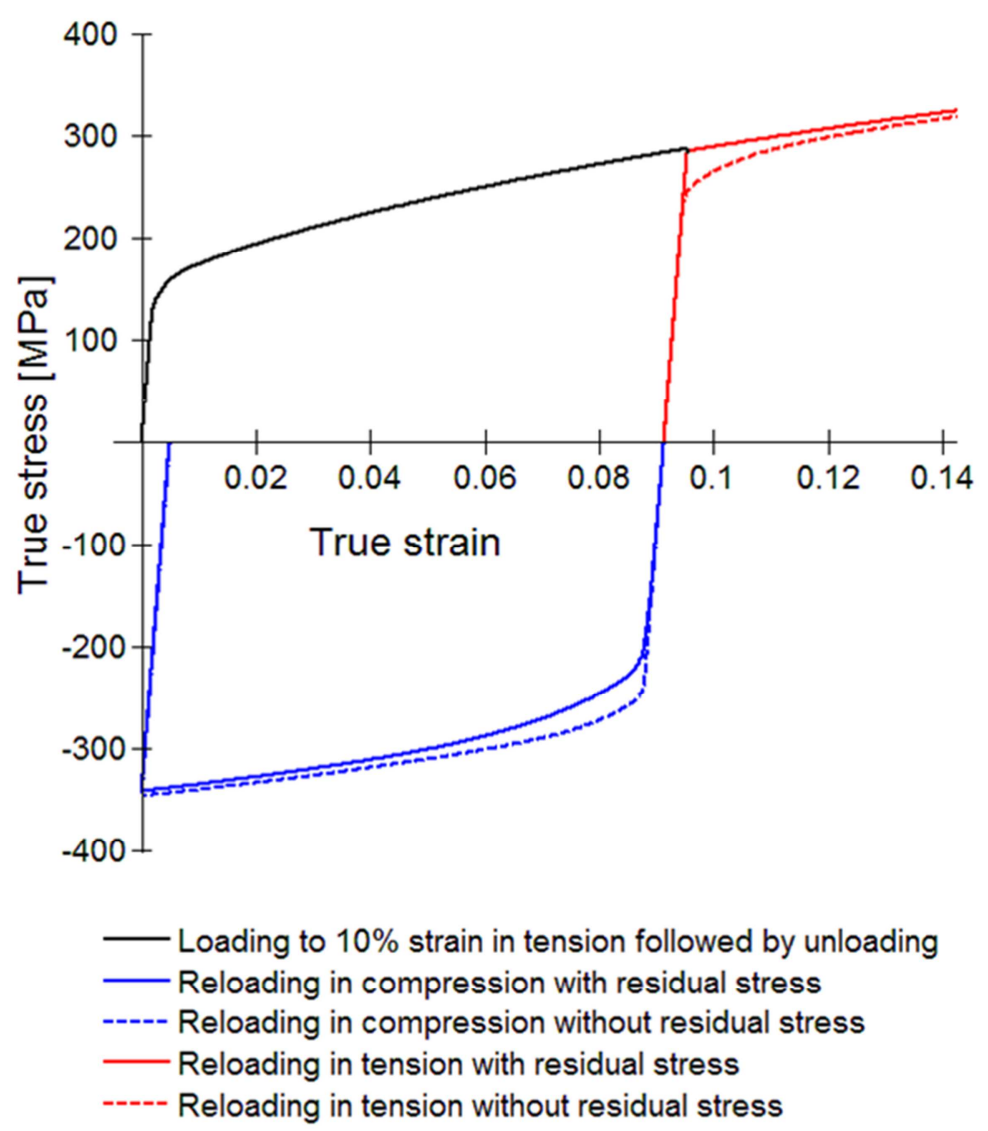

Fig. 6. Effect of residual stresses on material response upon reloading in forward and reverse directions.

\subsubsection{Effect of backstresses}

Figure $5 \mathrm{~b}$ compares measured stress-strain curves predicted with the model without consideration of the backstresses. To ensure as fair comparison as possible, the material parameters were readjusted. Table III shows the new material parameters used in the simulations without consideration of the backstresses. Evidently the backstresses are responsible for the early yielding upon reloading and rapid hardening that follows. These conclusions are in good agreement with prior works (Beyerlein and Tomé, 2007; Wilson and Bate, 1986; Wilson et al., 1990). In their analysis on $\mathrm{Cu}$ (Beyerlein and Tomé, 2007), the backstresses reached their maximum value between 0.05 and 0.1 true strain in forward loading. Upon the strain reversal, the backstresses are predicted to decay rapidly, ending at approximately 0.01 true strain (Beyerlein and Tomé, 2007). In the study done on low- and high-carbon steels in (Wilson and Bate, 1986), backstresses were estimated using the X-ray diffraction technique. Results of measurements showed that backstresses increase rapidly to approximately 0.03 true strain and continue to grow between 0.05 and 0.1 true strain with lower rate. After reversal the backstresses quickly decay and grow in the reverse direction reaching approximately $80 \%$ of their maximum value by 0.05 strain. Similarly to (Beyerlein and Tomé, 2007) they found that backstresses were nullified within 0.02 reverse strain. In our analysis, the model predicts that backstresses rapidly increase to approximately 0.05 true 
strain and then slowly saturate at approximately 0.15 true strain, while in the opposite direction the backstresses are erased by 0.01 true strain and rapidly grow.

Table III. Constitutive parameters for evolution of slip resistance (without backstresses).

\begin{tabular}{|l|l|l|l|l|}
\hline$\tau_{0}^{\alpha}[M P a]$ & $k_{1}^{\alpha}\left[m^{-1}\right]$ & $g^{\alpha}$ & $D^{\alpha}[M P a]$ & $q^{\alpha}$ \\
\hline 51 & $3.7 \times 10^{8}$ & 0.012 & 1500 & 16 \\
\hline
\end{tabular}

\subsubsection{Combined effect of residual stress and backstresses}

Figure 5c shows experimental results together with the EPSC calculations performed without consideration of the backstresses and residual stresses. These simulations were performed with the parameters reported in Table III. We observe that the non-linear unloading is not well captured, suggesting that the backstresses are mainly responsible for capturing the nonlinear unloading effects.

\subsubsection{Effect of reversible dislocations}

Figure 5d shows experimental results together with the EPSC calculations performed without consideration of reversible dislocation. The total dislocation density consisted of only forward dislocations. These simulations were performed with the parameters reported in Tables I and II. It is evident that without reversible dislocations the model is unable to predict the work hardening after reversal. It also should be noted that the effect of reversible dislocations on the predictions of the BE is minimal. Figure 7 shows a comparison between the evolutions of the total dislocation density during the cyclic tension-compression simulations in RD with and without consideration of the reversible dislocations. The results indicate that after the strain reversal a certain amount of plastic strain can be accumulated without a marked increase in total dislocation density. These results are in agreement with the constitutive assumption made to develop cyclic plasticity models in (Ohno, 1982; Ohno and Kachi, 1986).

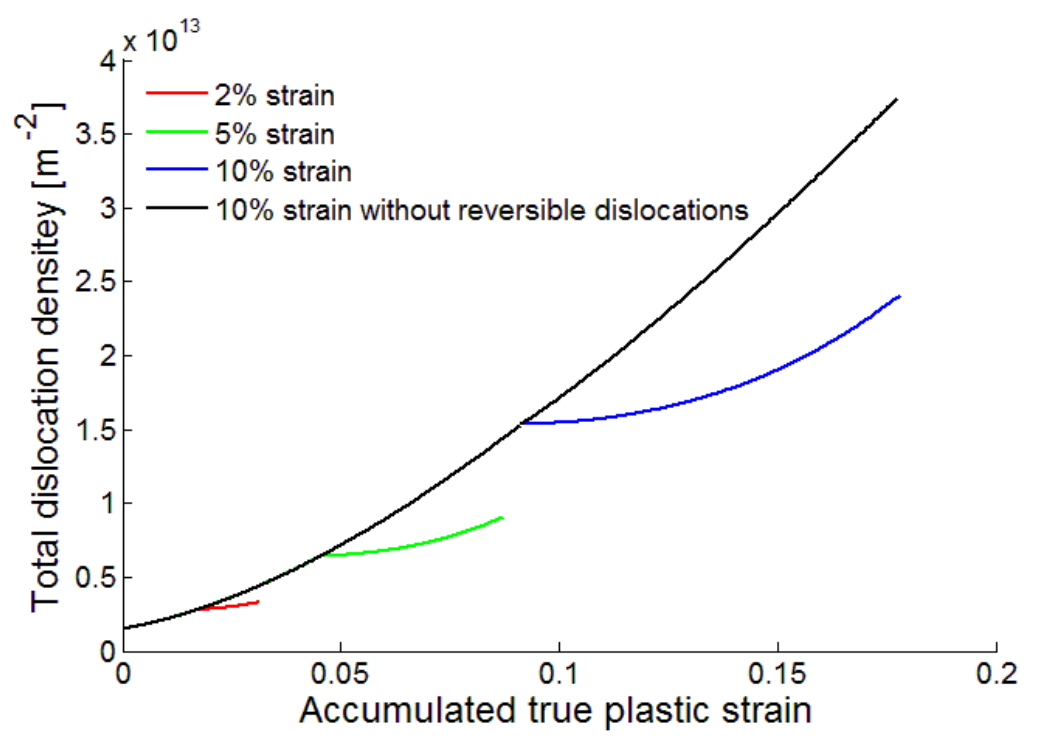


Fig. 7. Evolution of the total dislocation density during the cyclic tension-compression simulations in RD.

After the above analysis and interpretation, we can more confidently discuss the mechanism governing the cyclic response of the AA6022-T4 alloy. We infer first that the backstresses have a dominant effect on non-linear unloading, next that the inter-granular residual stresses and backstresses control the $\mathrm{BE}$, and finally that the inclusion of reversible dislocation motion is the key for capturing the hardening rates during reverse loading. As these effects have major implications on the accuracy of numerical simulations of forming processes, they must be captured by the constructive laws used in such simulations. The good predictions by the present model represent a significant incentive for incorporating the model into finiteelement (FE) frameworks to facilitate treatment of complex, non-monotonic deformation processes with heterogeneous boundary conditions. The resulting FE-EPSC model would couple sensitivity of microscopic mechanisms to complex macroscopic changes of imposed strain paths.

\section{Conclusions}

In this paper we presented a polycrystal plasticity model able to predict elasto-plastic cyclic tension-compression deformation behavior of metals to large plastic strain levels. The model is based on the self-consistent homogenization of single crystal responses and allows for a detailed comparison with macroscopic measurements. The performances of the developed model were tested on a data set collected for the AA6022-T4 sheets under in-plane cyclic deformation. The stress-strain response of the material exhibited the typical decreasing hardening rate in forward tensile deformation. The reverse deformation of the material started with linear and then non-linear unloading, followed by the transient Bauschinger effect, which is characterized by the early yielding and rapid increase in the work hardening rate. Finally, deformation during continuous loading in the reverse direction showed a decrease in the work hardening rate resulting in permanent softening. The latter indicated that the work hardening rate is larger during forward than during reverse loading.

To model the cyclic response of the material, the following main extensions to the EPSC model were implemented:

1. a kinematic hardening law at the slip system level able to capture the non-linear unloading behavior and the BE.

2. a dislocation density based hardening law that evolves the slip resistance while accounting for the forward and reverse glides. To predict the hardening behavior during loading in the reverse, the formulation models instantaneous rearrangement of the microstructure upon reloading, and progressive annihilation of dislocation during continuous loading in the reverse direction.

It was shown that the model successfully explains the flow response of the material during cyclic tension-compression deformation to large plastic pre-strains. Specifically, the plastic anisotropy, hardening rates, non-linear unloading, BE and permanent softening were simultaneously captured using a single set of material parameters. As many of these features 
in flow stress of AA6022-T4 are similar to those in other cubic metals, the present modeling framework is expected to be useful for a wider set of materials in addition to $\mathrm{Al}$ alloys.

\section{Acknowledgements}

This work is based upon a project supported by the National Science Foundation under grant CMMI-1301081. The authors are grateful to Z. Cedric Xia of Ford Motor Company for providing the cyclic tension-compression data of AA6022-T4. The EBSD work was performed in the electron microscopy laboratory at Los Alamos National Laboratory (LANL). The authors acknowledge Rodney J. McCabe of LANL for the EBSD characterization of the initial microstructure of the material. The authors also acknowledge numerous discussions with Professors Yannis P. Korkolis and Brad L. Kinsey of the University of New Hampshire.

\section{References}

Abel, A., 1987. Historical perspectives and some of the main features of the Bauschinger effect, Materials Forum. Institute of Metals and Materials Australasia, pp. 11-26.

Al-Harbi, H.F., Knezevic, M., Kalidindi, S.R., 2010. Spectral approaches for the fast computation of yield surfaces and first-order plastic property closures for polycrystalline materials with cubic-triclinic textures. CMC: Computers, Materials, \& Continua 15, 153172.

Alcoa, Alloy 6022 Sheet, North American Rolled Products

Ardeljan, M., Beyerlein, I.J., Knezevic, M., 2014a. A dislocation density based crystal plasticity finite element model: Application to a two-phase polycrystalline HCP/BCC composites. Journal of the Mechanics and Physics of Solids 66, 16-31.

Ardeljan, M., Knezevic, M., Nizolek, T., Beyerlein, I.J., Zheng, S.J., Carpenter, J.S., McCabe, R.J., Mara, N.A., Pollock, T.M., 2014b. A multi-scale model for texture development in $\mathrm{Zr} / \mathrm{Nb}$ nanolayered composites processed by accumulative roll bonding. IOP Conference Series: Materials Science and Engineering 63, 012170.

Armstrong, P.J., Frederick, C.O., 1966. A mathematical representation of multiaxial Bauschinger effect. Berkeley nuclear laboratories.

Barlat, F., Ferreira Duarte, J., Gracio, J., Lopes, A., Rauch, E., 2003. Plastic flow for nonmonotonic loading conditions of an aluminum alloy sheet sample. International Journal of Plasticity 19, 1215-1244.

Bate, P., Wilson, D., 1986a. Analysis of the Bauschinger effect. Acta Metallurgica 34, $1097-$ 1105.

Bate, P.S., Wilson, D.V., 1986b. Analysis of the bauschinger effect. Acta Metallurgica 34, 1097-1105.

Bauschinger, J., 1886. Über die Veränderung der Elasticitätsgrenze und Festigkeit des Eisen und Stahls durch Strecken und Quetschen, durch Erwarmen und Abkühlen und durch oftmal wiederholte Beanspruchung. Mitteilungen aus dem mechanisch-technischen Laboratorium der k. polytechnischen Schule, 1877-1836.

Beyerlein, I.J., McCabe, R.J., Tomé, C.N., 2011. Effect of microstructure on the nucleation of deformation twins in polycrystalline high-purity magnesium: A multi-scale modeling study. Journal of the Mechanics and Physics of Solids 59, 988-1003.

Beyerlein, I.J., Tomé, C.N., 2007. Modeling transients in the mechanical response of copper due to strain path changes. International Journal of Plasticity 23, 640-664.

Beyerlein, I.J., Tomé, C.N., 2008. A dislocation-based constitutive law for pure Zr including temperature effects. International Journal of Plasticity 24, 867-895. 
Bhattacharyya, A., Knezevic, M., Abouaf, M., 2015. Characterization of Crystallographic Texture and Intra-Grain Morphology in Cross-Rolled Tantalum. Metallurgical and Materials Transactions A 46, 1085-1096.

Boger, R., Wagoner, R., Barlat, F., Lee, M., Chung, K., 2005. Continuous, large strain, tension/compression testing of sheet material. International Journal of Plasticity 21, 23192343.

Brown, D.W., Beyerlein, I.J., Sisneros, T.A., Clausen, B., Tomé, C.N., 2012. Role of twinning and slip during compressive deformation of beryllium as a function of strain rate. International Journal of Plasticity 29, 120-135.

Brown, G.M., 1970. Inelastic deformation of an aluminum alloy under combined stress at elevated temperature. Journal of the Mechanics and Physics of Solids 18, 383-396.

Brown, L.M., Clarke, D.R., 1975. Work hardening due to internal stresses in composite materials. Acta Metallurgica 23, 821-830.

Buckley, S.N., Entwistle, K.M., 1956. The bauschinger effect in super-pure aluminum single crystals and polycrystals. Acta Metallurgica 4, 352-361.

Chaboche, J., Rousselier, G., 1983. On the plastic and viscoplastic constitutive equationsPart I: Rules developed with internal variable concept. Journal of Pressure Vessel Technology 105, 153-158.

Chaboche, J.L., 1977. Viscoplastic constitutive equations for the description of cyclic and ansiotropic behavior of metals, In: XVIIth Polish solid mechanics conference, Bulletin de l'Académie Polonaise des Sciences, Série Sciences et Techniques, pp. 33-41.

Chaboche, J.L., 2008. A review of some plasticity and viscoplasticity constitutive theories. International Journal of Plasticity 24, 1642-1693.

Chen, Z., Maekawa, S., Takeda, T., 1999. Bauschinger effect and multiaxial yield behavior of stress-reversed mild steel. Metallurgical and Materials Transactions A 30, 3069-3078.

Christodoulou, N., Woo, O.T., MacEwen, S.R., 1986. Effect of stress reversals on the work hardening behaviour of polycrystalline copper. Acta Metallurgica 34, 1553-1562.

Chun, B.K., Jinn, J.T., Lee, J.K., 2002. Modeling the Bauschinger effect for sheet metals, part I: theory. International Journal of Plasticity 18, 571-595.

Cleveland, R.M., Ghosh, A.K., 2002. Inelastic effects on springback in metals. International Journal of Plasticity 18, 769-785.

Demir, E., Raabe, D., 2010. Mechanical and microstructural single-crystal Bauschinger effects: Observation of reversible plasticity in copper during bending. Acta Materialia 58, 6055-6063.

Dietrich, L., Turski, K., 1978. New method of sheet metal compression tests. Rozp. Inz. 26.

Eshelby, J.D., 1957. The determination of the elastic field of an ellipsoidal inclusion, and related problems. Proc R. Soc. Lond. A 241, 376-396.

Fast, T., Knezevic, M., Kalidindi, S.R., 2008. Application of microstructure sensitive design to structural components produced from hexagonal polycrystalline metals. Computational Materials Science 43, 374-383.

Fromm, B.S., Adams, B.L., Ahmadi, S., Knezevic, M., 2009. Grain size and orientation distributions: Application to yielding of $\alpha$-titanium. Acta Materialia 57, 2339-2348.

Gough, H., Hanson, D., Wright, S., 1927. The Behaviour of Single Crystals of Aluminium under Static and Repeated Stresses. Philosophical Transactions of the Royal Society of London. Series A, Containing Papers of a Mathematical or Physical Character, 1-30.

Gracio, J.J., Barlat, F., Rauch, E.F., Jones, P.T., Neto, V.F., Lopes, A.B., 2004. Artificial aging and shear deformation behaviour of 6022 aluminium alloy. International Journal of Plasticity 20, 427-445.

Greetham, G., Honeycombe, R., 1960. The deformation of single crystals of aluminum 4.5percent copper alloy. Journal of the Institute of Metals 89, 13-21. 
Hart, E.W., 1972. Theory of dispersion hardening in metals. Acta Metallurgica 20, 275-289.

Hasegawa, T., Yakou, T., Karashima, S., 1975a. Deformation behaviour and dislocation structures upon stress reversal in polycrystalline aluminium. Materials Science and Engineering 20, 267-276.

Hasegawa, T., Yakou, T., Karashima, S., 1975b. Deformation behaviour and dislocation structures upon stress reversal in polycrystalline aluminium. Materials Science and Engineering 20, 267-276.

Hu, Z., Rauch, E.F., Teodosiu, C., 1992. Work-hardening behavior of mild steel under stress reversal at large strains. International Journal of Plasticity 8, 839-856.

Hutchinson, J., 1970. Elastic-plastic behaviour of polycrystalline metals and composites. Proceedings of the Royal Society of London. A. Mathematical and Physical Sciences 319, 247-272.

Jackson, P.J., 1985. Dislocation modelling of shear in f.c.c. crystals. Progress in Materials Science 29, 139-175.

Jahedi, M., Knezevic, M., Paydar, M., 2015a. High-Pressure Double Torsion as a Severe Plastic Deformation Process: Experimental Procedure and Finite Element Modeling. J. of Materi Eng and Perform 24, 1471-1482.

Jahedi, M., Paydar, M.H., Knezevic, M., 2015b. Enhanced microstructural homogeneity in metal-matrix composites developed under high-pressure-double-torsion. Materials Characterization 104, 92-100.

Jahedi, M., Paydar, M.H., Zheng, S., Beyerlein, I.J., Knezevic, M., 2014. Texture evolution and enhanced grain refinement under high-pressure-double-torsion. Materials Science and Engineering: A 611, 29-36.

Kalidindi, S.R., Bronkhorst, C.A., Anand, L., 1992. Crystallographic Texture Evolution in Bulk Deformation Processing of Fcc Metals. Journal of the Mechanics and Physics of Solids 40, 537-569.

Kalidindi, S.R., Duvvuru, H.K., Knezevic, M., 2006. Spectral calibration of crystal plasticity models. Acta Materialia 54, 1795-1804.

Kalidindi, S.R., Knezevic, M., Niezgoda, S., Shaffer, J., 2009. Representation of the orientation distribution function and computation of first-order elastic properties closures using discrete Fourier transforms. Acta Materialia 57, 3916-3923.

Kassner, M.E., Geantil, P., Levine, L.E., 2013. Long range internal stresses in single-phase crystalline materials. International Journal of Plasticity 45, 44-60.

Kim, J.H., Kim, D., Barlat, F., Lee, M.-G., 2012. Crystal plasticity approach for predicting the Bauschinger effect in dual-phase steels. Materials Science and Engineering: A 539, 259-270.

Kitayama, K., Tomé, C.N., Rauch, E.F., Gracio, J.J., Barlat, F., 2013. A crystallographic dislocation model for describing hardening of polycrystals during strain path changes. Application to low carbon steels. International Journal of Plasticity in press.

Knezevic, M., Al-Harbi, H.F., Kalidindi, S.R., 2009. Crystal plasticity simulations using discrete Fourier transforms. Acta Materialia 57, 1777-1784.

Knezevic, M., Beyerlein, I.J., Brown, D.W., Sisneros, T.A., Tomé, C.N., 2013a. A polycrystal plasticity model for predicting mechanical response and texture evolution during strain-path changes: Application to beryllium. International Journal of Plasticity 49, 185-198.

Knezevic, M., Beyerlein, I.J., Lovato, M.L., Tomé, C.N., Richards, A.W., McCabe, R.J., 2014a. A strain-rate and temperature dependent constitutive model for BCC metals incorporating non-Schmid effects: Application to tantalum-tungsten alloys. International Journal of Plasticity 62, 93-104. 
Knezevic, M., Beyerlein, I.J., Nizolek, T., Mara, N.A., Pollock, T.M., 2013b. Anomalous Basal Slip Activity in Zirconium under High-strain Deformation. Materials Research Letters 1, 133-140.

Knezevic, M., Capolungo, L., Tomé, C.N., Lebensohn, R.A., Alexander, D.J., Mihaila, B., McCabe, R.J., 2012. Anisotropic stress-strain response and microstructure evolution of textured $\alpha$-uranium. Acta Materialia 60, 702-715.

Knezevic, M., Carpenter, J.S., Lovato, M.L., McCabe, R.J., 2014b. Deformation behavior of the cobalt-based superalloy Haynes 25: Experimental characterization and crystal plasticity modeling. Acta Materialia 63, 162-168.

Knezevic, M., Drach, B., Ardeljan, M., Beyerlein, I.J., 2014c. Three dimensional predictions of grain scale plasticity and grain boundaries using crystal plasticity finite element models. Computer Methods in Applied Mechanics and Engineering 277, 239-259.

Knezevic, M., Jahedi, M., Korkolis, Y.P., Beyerlein, I.J., 2014d. Material-based design of the extrusion of bimetallic tubes. Computational Materials Science 95, 63-73.

Knezevic, M., Kalidindi, S.R., 2007. Fast computation of first-order elastic-plastic closures for polycrystalline cubic-orthorhombic microstructures. Computational Materials Science 39, 643-648.

Knezevic, M., Kalidindi, S.R., Fullwood, D., 2008a. Computationally efficient database and spectral interpolation for fully plastic Taylor-type crystal plasticity calculations of facecentered cubic polycrystals. International Journal of Plasticity 24, 1264-1276.

Knezevic, M., Kalidindi, S.R., Mishra, R.K., 2008b. Delineation of first-order closures for plastic properties requiring explicit consideration of strain hardening and crystallographic texture evolution. International Journal of Plasticity 24, 327-342.

Knezevic, M., Landry, N.W., 2015. Procedures for reducing large datasets of crystal orientations using generalized spherical harmonics. Mechanics of Materials, doi:10.1016/j.mechmat.2015.04.014.

Knezevic, M., Lebensohn, R.A., Cazacu, O., Revil-Baudard, B., Proust, G., Vogel, S.C., Nixon, M.E., 2013c. Modeling bending of $\alpha$-titanium with embedded polycrystal plasticity in implicit finite elements. Materials Science and Engineering: A 564, 116-126.

Knezevic, M., Levinson, A., Harris, R., Mishra, R.K., Doherty, R.D., Kalidindi, S.R., 2010. Deformation twinning in AZ31: Influence on strain hardening and texture evolution. Acta Materialia 58, 6230-6242.

Knezevic, M., McCabe, R.J., Lebensohn, R.A., Tomé, C.N., Liu, C., Lovato, M.L., Mihaila, B., 2013d. Integration of self-consistent polycrystal plasticity with dislocation density based hardening laws within an implicit finite element framework: Application to lowsymmetry metals. Journal of the Mechanics and Physics of Solids 61, 2034-2046.

Knezevic, M., McCabe, R.J., Tomé, C.N., Lebensohn, R.A., Chen, S.R., Cady, C.M., Gray Iii, G.T., Mihaila, B., 2013e. Modeling mechanical response and texture evolution of $\alpha-$ uranium as a function of strain rate and temperature using polycrystal plasticity. International Journal of Plasticity 43, 70-84.

Knezevic, M., Nizolek, T., Ardeljan, M., Beyerlein, I.J., Mara, N.A., Pollock, T.M., 2014e. Texture evolution in two-phase $\mathrm{Zr} / \mathrm{Nb}$ lamellar composites during accumulative roll bonding. International Journal of Plasticity 57, 16-28.

Knezevic, M., Savage, D.J., 2014. A high-performance computational framework for fast crystal plasticity simulations. Computational Materials Science 83, 101-106.

Knezevic, M., Zecevic, M., Beyerlein, I.J., Bingert, J.F., McCabe, R.J., 2015. Strain rate and temperature effects on the selection of primary and secondary slip and twinning systems in HCP Zr. Acta Materialia 88, 55-73.

Kocks, U.F., Mecking, H., 1981. Kinetics of Flow and Strain-Hardening. Acta Metallurgica 29, 1865-1875. 
Kocks, U.F., Mecking, H., 2003. Physics and phenomenology of strain hardening: the FCC case. Progress in Materials Science 48, 171-273.

Kuhlmann-Wilsdorf, D., 1989. Theory of plastic deformation:-properties of low energy dislocation structures. Materials Science and Engineering: A 113, 1-41.

Lavrentev, F.F., 1980. The type of dislocation interaction as the factor determining work hardening. Materials Science and Engineering 46, 191-208.

Lebensohn, R.A., Tomé, C.N., 1993. A self-consistent anisotropic approach for the simulation of plastic deformation and texture development of polycrystals: Application to zirconium alloys. Acta Metallurgica et Materialia 41, 2611-2624.

Lentz, M., Klaus, M., Beyerlein, I.J., Zecevic, M., Reimers, W., Knezevic, M., 2015a. In situ $\mathrm{X}$-ray diffraction and crystal plasticity modeling of the deformation behavior of extruded $\mathrm{Mg}-\mathrm{Li}$-(Al) alloys: An uncommon tension-compression asymmetry. Acta Materialia 86, 254-268.

Lentz, M., Klaus, M., Wagner, M., Fahrenson, C., Beyerlein, I.J., Zecevic, M., Reimers, W., Knezevic, M., 2015b. Effect of age hardening on the deformation behavior of an $\mathrm{Mg}-\mathrm{Y}-$ Nd alloy: In-situ X-ray diffraction and crystal plasticity modeling. Materials Science and Engineering: A 628, 396-409.

Li, K., Carden, W., Wagoner, R., 2002. Simulation of springback. International Journal of Mechanical Sciences 44, 103-122.

Li, L., Shen, L., Proust, G., 2014. A texture-based representative volume element crystal plasticity model for predicting Bauschinger effect during cyclic loading. Materials Science and Engineering: A 608, 174-183.

Li, Y., Aubin, V., Rey, C., Bompard, P., 2012. Polycrystalline numerical simulation of variable amplitude loading effects on cyclic plasticity and microcrack initiation in austenitic steel 304L. International Journal of Fatigue 42, 71-81.

Lopes, A.B., Rauch, E.F., Gracio, J.J., 1999. Textural vs structural plastic instabilities in sheet metal forming. Acta Materialia 47, 859-866.

Lorentzen, T., Daymond, M.R., Clausen, B., Tomé, C.N., 2002. Lattice strain evolution during cyclic loading of stainless steel. Acta materialia 50, 1627-1638.

Madec, R., Devincre, B., Kubin, L., Hoc, T., Rodney, D., 2003. The role of collinear interaction in dislocation-induced hardening. Science 301, 1879-1882.

McDowell, D.L., 1992. A nonlinear kinematic hardening theory for cyclic thermoplasticity and thermoviscoplasticity. International Journal of Plasticity 8, 695-728.

Mecking, H., Kocks, U.F., 1981. Kinetics of flow and strain-hardening. Acta Metallurgica et Materialia 29, 1865-1875.

Meyers M.A., C.K.K., 2009. Mechanical behavior of materials, Cambridge.

Miao, W.F., Laughlin, D.E., 1999. Precipitation hardening in aluminum alloy 6022. Scripta Materialia 40, 873-878.

Mihaila, B., Knezevic, M., Cardenas, A., 2014. Three orders of magnitude improved efficiency with high-performance spectral crystal plasticity on GPU platforms. International Journal for Numerical Methods in Engineering 97, 785-798.

Mompiou, F., Caillard, D., Legros, M., Mughrabi, H., 2012. In situ TEM observations of reverse dislocation motion upon unloading in tensile-deformed UFG aluminium. Acta Materialia 60, 3402-3414.

Mughrabi, H., 1983. Dislocation wall and cell structures and long-range internal stresses in deformed metal crystals. Acta Metallurgica 31, 1367-1379.

Neil, C.J., Wollmershauser, J.A., Clausen, B., Tome, C.N., Agnew, S.R., Modeling lattice strain evolution at finite strains and experimental verification for copper and stainless steel using in situ neutron diffraction. International Journal of Plasticity 26, 1772-1791. 
Nieh, T.G., Nix, W.D., 1986. Unloading yield effects in aluminum alloys. Metallurgical transactions. A, Physical metallurgy and materials science 17 A, 121-126.

Ohno, N., 1982. A Constitutive Model of Cyclic Plasticity With a Nonhardening Strain Region. J. Appl. Mech. 49(4), 721-727

Ohno, N., Kachi, Y., 1986. A Constitutive Model of Cyclic Plasticity for Nonlinear Hardening Materials. J. Appl. Mech. 53(2), 395-403.

Orowan, E., 1959. Causes and Effects of Internal Stresses, General Motors Symposium, ed. G.M. Rassweiler and W.L. Grube. Elsevier, Amsterdam, pp. 59-80.

Pavlina, E., Lee, M.-G., Barlat, F., 2015. Observations on the Nonlinear Unloading Behavior of Advanced High Strength Steels. Metallurgical and Materials Transactions A 46, 18-22.

Peeters, B., Bacroix, B., Teodosiu, C., Van Houtte, P., Aernoudt, E., 2001a. Workhardening/softening behaviour of b.c.c. polycrystals during changing strain paths: II. TEM observations of dislocation sheets in an IF steel during two-stage strain paths and their representation in terms of dislocation densities. Acta Materialia 49, 1621-1632.

Peeters, B., Seefeldt, M., Teodosiu, C., Kalidindi, S.R., Van Houtte, P., Aernoudt, E., 2001b. Work-hardening/softening behaviour of b.c.c. polycrystals during changing strain paths: I. An integrated model based on substructure and texture evolution, and its prediction of the stress-strain behaviour of an IF steel during two-stage strain paths. Acta Materialia 49, 1607-1619.

Proudhon, H., Poole, W., Wang, X., Brechet, Y., 2007. The Bauschinger effect and internal stresses in 6111 Aluminium alloys. Philosophical Magazine.

Rauch, E.F., 1997. The stresses and work hardening rates of mild steel with different dislocation patterns. Materials Science and Engineering: A 234-236, 653-656.

Rauch, E.F., Gracio, J.J., Barlat, F., 2007. Work-hardening model for polycrystalline metals under strain reversal at large strains. Acta Materialia 55, 2939-2948.

Sachs, G., 1929. Zur ableitung einer fliessbedingung, Mitteilungen der deutschen Materialprüfungsanstalten. Springer, pp. 94-97.

Sauzay, M., 2008. Analytical modelling of intragranular backstresses due to deformation induced dislocation microstructures. International Journal of Plasticity 24, 727-745.

Seeger, A., 1957. Glide and work hardening in face-centered cubic and hexagonal closepacked metals, in: Fisher, J.C. (Ed.), Dslocations and Mechanical Properties of Cryslals, Wiley, New York, p. p.243.

Shaffer, J.B., Knezevic, M., Kalidindi, S.R., 2010. Building texture evolution networks for deformation processing of polycrystalline fcc metals using spectral approaches: Applications to process design for targeted performance. International Journal of Plasticity 26, 1183-1194.

Smith, A., Chen, Z., Lee, J.Y., Lee, M.G., Wagoner, R.H., 2014. Effective method for fitting complex constitutive equations. International Journal of Plasticity 58, 100-119.

Sritharan, T., Chandel, R.S., 1997. Phenomena in interrupted tensile tests of heat treated aluminium alloy 6061. Acta Materialia 45, 3155-3161.

Stout, M., Rollett, A., 1990. Large-strain Bauschinger effects in fcc metals and alloys. Metallurgical and Materials Transactions A 21, 3201-3213.

Szczepiński, W., 1990. Experimental methods in mechanics of solids. Elsevier

Tan, Z., Magnusson, C., Persson, B., 1994. The Bauschinger effect in compression-tension of sheet metals. Materials Science and Engineering: A 183, 31-38.

Tanaka, K., Mori, T., 1970. The hardening of crystals by non-deforming particles and fibres. Acta Metallurgica 18, 931-941.

Taylor, G.I., 1938. Plastic strain in metals. Journal of the Institute of Metals 62, 307-324.

Trivedi, P., Field, D.P., Weiland, H., 2004. Alloying effects on dislocation substructure evolution of aluminum alloys. International Journal of Plasticity 20, 459-476. 
Turner, P.A., Tomé, C.N., 1994. A study of residual stresses in Zircaloy-2 with rod texture. Acta Metallurgica et Materialia 42, 4143-4153.

Wagoner, R.H., Lim, H., Lee, M.-G., 2013. Advanced Issues in springback. International Journal of Plasticity 45, 3-20.

Wang, Z., Beyerlein, I., LeSar, R., 2007. The importance of cross-slip in high-rate deformation. Modelling and Simulation in Materials Science and Engineering 15, 675.

Wen, W., Borodachenkova, M., Tomé, C.N., Vincze, G., Rauch, E.F., Barlat, F., Grácio, J.J., Mechanical behavior of $\mathrm{Mg}$ subjected to strain path changes: Experiments and modeling. International Journal of Plasticity.

Weng, G.J., 1979. Kinematic hardening rule in single crystals. International Journal of Solids and Structures 15, 861-870.

Weng, G.J., 1980. Constitutive equations of single crystals and polycrystalline aggregates under cyclic loading. International Journal of Engineering Science 18, 1385-1397.

Wilson, D.V., Bate, P.S., 1986. Reversibility in the work hardening of spheroidised steels. Acta Metallurgica 34, 1107-1120.

Wilson, D.V., Zandrahimi, M., Roberts, W.T., 1990. Effects of changes in strain path on work-hardening in $\mathrm{CP}$ aluminium and an $\mathrm{Al}-\mathrm{Cu}-\mathrm{Mg}$ alloy. Acta Metallurgica et Materialia 38, 215-226.

Wollmershauser, J.A., Clausen, B., Agnew, S.R., 2012. A slip system-based kinematic hardening model application to in situ neutron diffraction of cyclic deformation of austenitic stainless steel. International Journal of Fatigue 36, 181-193.

Wu, P.D., MacEwen, S.R., Lloyd, D.J., Jain, M., Tugcu, P., Neale, K.W., 2005. On prestraining and the evolution of material anisotropy in sheet metals. International Journal of Plasticity 21, 723-739.

Wu, X., Proust, G., Knezevic, M., Kalidindi, S.R., 2007. Elastic-plastic property closures for hexagonal close-packed polycrystalline metals using first-order bounding theories. Acta Materialia 55, 2729-2737.

$\mathrm{Xu}$, B., Jiang, Y., 2004. A cyclic plasticity model for single crystals. International Journal of Plasticity 20, 2161-2178.

Yoshida, F., Uemori, T., Fujiwara, K., 2002. Elastic-plastic behavior of steel sheets under inplane cyclic tension-compression at large strain. International Journal of Plasticity 18, 633-659.

Zang, S.-1., Lee, M.-g., Hoon Kim, J., 2013. Evaluating the significance of hardening behavior and unloading modulus under strain reversal in sheet springback prediction. International Journal of Mechanical Sciences 77, 194-204.

Zecevic, M., Knezevic, M., Beyerlein, I.J., Tomé, C.N., 2015a. An elasto-plastic selfconsistent model with hardening based on dislocation density, twinning and de-twinning: Application to strain path changes in HCP metals. Materials Science and Engineering: A 638, 262-274.

Zecevic, M., McCabe, R.J., Knezevic, M., 2015b. A new implementation of the spectral crystal plasticity framework in implicit finite elements. Mechanics of Materials 84, 114126.

Zecevic, M., McCabe, R.J., Knezevic, M., 2015c. Spectral database solutions to elastoviscoplasticity within finite elements: Application to a cobalt-based FCC superalloy. International Journal of Plasticity 70, 151-165.

Zhou, C., LeSar, R., 2012. Dislocation dynamics simulations of the Bauschinger effect in metallic thin films. Computational Materials Science 54, 350-355. 
A new elasto-plastic polycrystal model for the prediction of cyclic deformation is developed.

The model considers forward and reverse dislocation glide in evolving slip resistance.

The model accounts for the inter-granular stresses and intra-granular backstresses.

Anisotropy, hardening, non-linear unloading, Bauschinger effect are simultaneously predicted. 